\title{
The Classification of Monopoles for the Classical Groups
}

\author{
Jacques Hurtubise* \\ Institute for Advanced Study Princeton, NJ 08540, USA and Department of Mathematics, \\ McGill University, Burnside Hall, 805 Sherbrooke St. W. Montréal, Québec, Canada H3A 2K6
}

\begin{abstract}
By studying a construction of $\mathrm{Nahm}$, we compute the moduli spaces of monopoles with maximal symmetry breaking at infinity for $S U(N), S O(N)$ and $S p(N)$; these are found to be equivalent to spaces of holomorphic maps from $\mathbb{P}_{1}$ into flag manifolds.
\end{abstract}

\section{Introduction}

Let $P$ be a principal $G$-bundle over $\mathbb{R}^{3}, G$ a compact group, $\nabla$ a connection on $P$ with curvature $F, \varphi$ (the "Higgs field") a section of $\operatorname{ad}(P)$, the associated adjoint bundle: $(\nabla, \varphi)$ is a monopole if it solves the Bogomoln'yi equation, $F=* \nabla \varphi$, and if it satisfies the boundary condition of having finite action, with $\varphi$ tending toward a finite limit at infinity, with values in a fixed $G$-orbit in $\operatorname{ad}(P)$. Such monopoles, particularly for the group $S U(2)$, have been extensively studied in recent years, from various points of view [JT, Hi, Mu]. One particularly successful construction, due to Nahm [N], describes these monopoles in terms of solutions to some non-linear ordinary differential equations, Nahm's equations. A theorem, whose full proof is due to Hitchin [Hi], shows that for $S U(2)$, there is a natural equivalence between $S U(2)$ monopoles and an appropriate class of solutions to Nahm's equations. Using this, Donaldson was able to give a description of the moduli space of $S U(2)$ monopoles:

Theorem [D1]. Given an isomorphism $\mathbb{R}^{3} \cong \mathbb{R} \times \mathbb{C}$, compatible with the usual metrics there is a natural correspondence between a circle bundle $\tilde{M}_{k}$ defined over the moduli space of $S U(2)$ monopoles of charge $k$, and the complex manifold $R_{k}$ of rational maps $f: \mathbb{P}_{1} \rightarrow \mathbb{P}_{1}$ of degree $k$, with $f(\infty)=0$.

In terms of the monopole, the extra circle corresponds to the choice of a framing at infinity; see [AHi, Hu].

Recently, in [HuM], a proof was given of the validity of Nahm's construction for all the classical groups, for monopoles with maximal symmetry breaking at infinity. This condition means that if $G$ is the gauge group with maximal torus $T$,

* Research supported in part by NSERC grant A8361 and FCAR grant EQ3518 
the Higgs field at infinity takes values in a fixed adjoint orbit of the form $G / T$. These monopoles have a discrete classification given by their "topological charge." an element of $\pi_{2}(G / T)$ defined in terms of their Higgs field at infinity. This charge is essentially an $r$-tuple $\left(m_{1}, \ldots, m_{r}\right)$ of integers, where $r=\operatorname{rank}(G)$; it can be shown that, once suitable normalizations are made, $m_{i} \geqq 0$. For $S U(N)$ the result is then:

Theorem [N, HuM]. There is a natural correspondence between:

A) Elements of a non-empty union $M_{m}=M\left(m_{1}, \ldots, m_{N-1}\right)$ of connected components of the moduli space of $S U(N)$ monopoles of charge $m=\left(m_{1}, \ldots, m_{N-1}\right)$ with $\varphi$ asymptotic to a conjugate of $\operatorname{diag}\left(\mu_{1}, \ldots, \mu_{N}\right), \mu_{1}<\cdots<\mu_{N}$, and

B) Conjugacy classes under $U\left(m_{j}\right)$, for each non-zero $m_{j}$, of analytic $u\left(m_{j}\right)$-valued functions ${ }^{i} T_{j}(z), i=1,2,3$, on the intervals $\left(\mu_{j}, \mu_{j+1}\right)$, such that,

1) The ${ }^{i} T_{j}$ solve Nahm's equations

$$
\frac{d^{i} T_{j}}{d z}+\frac{1}{2} \sum_{k, l=1}^{3} \varepsilon_{i k l}\left[{ }^{k} T_{j},{ }^{l} T_{j}\right]=0 .
$$

2) At a boundary point $\mu_{j}$, setting $t=\left(z-\mu_{j}\right)$, with the convention $m_{0}=m_{N}=0$,

i) if $m_{j-1}<m_{j}$, - there exist finite, non-zero limits $C_{j}^{i}=\lim _{t \rightarrow 0^{-}}{ }^{i} T_{j-1}(t)$ and ${ }^{i} T_{j-1}(t)$ is analytic at
$t=0$.

-For $t>0$, one can conjugate ${ }^{i} T_{j}(t)$ by a unitary matrix so that one has the expansion near $t=0$ :

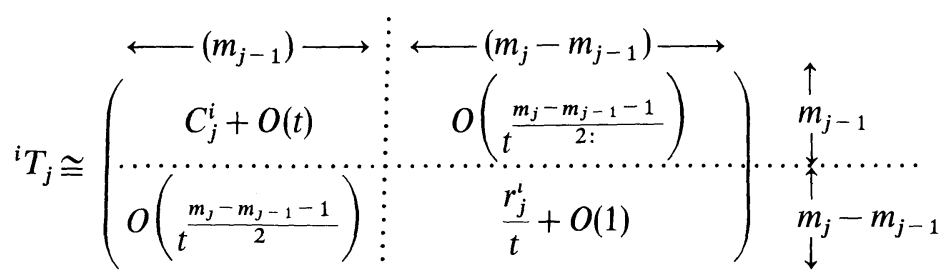

The upper diagonal block is analytic in $t$; the lower diagonal block is meromorphic in $t$, and the off-diagonal blocks are of the form $t^{\left.\frac{\left(m_{j}-m_{j-1}-1\right.}{2}\right)} \times($ analytic in $t)$.

ii) if $m_{j-1}>m_{j}$,

one has the same boundary behaviour, but with the roles of $\left(\mu_{j-1}, \mu_{j}\right),\left(\mu_{j}, \mu_{j+1}\right)$ reversed.

iii) if $m_{j-1}=m_{j}$,

one has finite analytic limits $C_{i}^{+}, C_{i}^{-}$, of the ${ }^{i} T$ from both sides of $\mu_{j}$; if one sets

$$
A^{ \pm}(\zeta)=\left(C_{2}^{ \pm}+i C_{3}^{ \pm}\right)+\left(2 i C_{1}^{ \pm}\right) \zeta+\left(C_{2}^{ \pm}-i C_{3}^{ \pm}\right) \zeta^{2},
$$

one asks that $A^{+}(\zeta)-A^{-}(\zeta)$ be of rank at most one for all $\zeta$.

3) For $m_{j-1} \neq m_{j}$ the residues $r_{j}^{i}$ define an irreducible representation of $s u(2)$.

For the groups $S O(N), S p(k)$ one embeds $S O(N)$ into $S U(N), S p(k)$ into $S U(2 k)$ 
in the natural way. One then has the following table.

\begin{tabular}{|c|c|c|c|c|}
\hline $\begin{array}{l}\text { A } G \text {-monopole } \\
\text { for } G=\end{array}$ & $\begin{array}{l}\text { with } \\
G \text {-charges: }\end{array}$ & $\begin{array}{l}\text { embedded } \\
\text { in } S U(N)\end{array}$ & $\begin{array}{l}\text { As an } S U(N) \text { - } \\
\text { monopole, its Higgs } \\
\text { field is asymptotic } \\
\text { to } \operatorname{diag}\left(\mu_{\imath}\right) \text { with: }\end{array}$ & $\begin{array}{l}\text { and is has } S U(N) \\
\text { charges } m_{i} \text {, with }\end{array}$ \\
\hline$S p(k)$ & $\rho_{1}, \ldots, \rho_{k}$ & $N=2 k$ & $\begin{aligned} \mu_{i} & =-\mu_{2 k+1-i} \\
i & =1, \ldots, k\end{aligned}$ & $\begin{array}{c}m_{l}=m_{2 k-i}=\rho_{i} \\
i=1, \ldots, k\end{array}$ \\
\hline$S O(2 k)$ & $\begin{array}{l}\rho_{1}, \ldots, \rho_{k-2} \\
\rho_{+}, \rho_{-}\end{array}$ & $N=2 k$ & $\begin{aligned} \mu_{i} & =-\mu_{2 k+1-\imath} \\
i & =1, \ldots, k\end{aligned}$ & $\begin{array}{l}m_{i}=m_{2 k-i}=\rho_{i} \\
\quad i=1, \ldots, k-2 \\
m_{k-1}=m_{k+1}=\rho_{+}+\rho_{-} \\
\quad m_{k}=2 \rho_{+} .\end{array}$ \\
\hline$S O(2 k+1)$ & $\rho_{1}, \ldots, \rho_{k}$ & $N=2 k+1$ & $\begin{aligned} \mu_{i} & =-\mu_{2 k+2-i} \\
i & =1, \ldots, k+1\end{aligned}$ & $\begin{aligned} m_{i} & =m_{2 k+1-i}=\rho_{i} \\
i & =1, \ldots, k-1 \\
m_{k} & =m_{k+1}=2 \rho_{k}\end{aligned}$ \\
\hline
\end{tabular}

With this in mind, one then has

Theorem [N, HuM]. There is a natural equivalence between

A) Elements of a non-empty union $M_{\rho}$ of components of the moduli space of $G$-monopoles of charge $\rho$, with $\varphi$ asymptotic under the inclusion of $G$ into $S U(N)$ to a conjugate of $\operatorname{diag}\left(\mu_{j}\right), \mu_{1}<\cdots<\mu_{N}$, and

B) Conjugacy classes under $U\left(m_{j}\right)$ for the $m_{j} \neq 0$, of analytic $u\left(m_{j}\right)$-valued functions ${ }^{i} T_{j}(z)$ on $\left(\mu_{j}, \mu_{j+1}\right) i=1,2,3$, satisfying conditions:

1), 2), 3) as above, and

4) There are matrices $c_{j}$ with

$$
{ }^{i} T_{N-j+1}(-z)^{T}=\left(c_{j}\right)\left({ }^{i} T_{j}(z)\right)\left(c_{j}\right)^{-1},
$$

$c_{j}, c_{j-1}$ being compatible in the obvious way at the boundary points, and with

$$
c_{N-j+1}=c_{j}^{T} \text { for } \quad S p, \quad c_{N-j+1}=-c_{j}^{T} \text { for } \quad \text { SO. }
$$

Remark. $M_{m}$ is essentially the union of the components of the moduli space containing multi-monopoles looking like the sum of simple monopoles spaced far apart; we shall see later that $M_{m}$ is in fact connected. It is widely expected that the monopole moduli itself is connected: this is in fact the case for $S U(2)$ and $S U(3)$ [T]. If so, then the theorem above applies to all monopoles.

Once this correspondence with solutions to Nahm's equations is proven, it is natural to try to emulate Donaldson and describe the space $M_{\rho}$. This is the purpose of this article; we prove a conjecture of Atiyah and Murray:

Theorem. Let $G$ be a classical compact group ( $S U(n), S p(n)$, $S O(n))$; let $T$ be a maximal torus of $G$ and $g_{0}$ a fixed element of $G$.

Given an isomorphism $\mathbb{R}^{3} \sim \mathbb{R} \times \mathbb{C}$, compatible with the usual matrics, there is a space $\tilde{M}_{\rho}$ mapping to $M_{\rho}$, which over the open set in $M_{\rho}$ of irreducible monopoles is a principal T-bundle, and a natural bijective correspondence between $\tilde{M}_{\rho}$ and the 
complex variety $R_{\rho}$ of rational holomorphic maps

$$
f: \mathbb{P}_{1} \rightarrow G / T
$$

of degree $\rho$ such that $f(\infty)=g_{0} T$.

The fibre of $\tilde{M}_{\rho}$ over $M_{\rho}$ corresponds to a choice of framing of the monopole at infinity; see Sect. 4.

The strategy of the proof follows that of Donaldson:

-One first divides Nahm's equations into a "real" equation and a "complex" equation, in such a way that the complex equation is invariant under a complex group $\mathscr{G}$ of gauge transformations; one then shows by variational methods that each $\mathscr{G}$-orbit contains an essentially unique solution to the real equation.

-One then classifies the $\mathscr{G}$-orbits in terms of rational maps. Essentially, we will find that the algebraic data we can extract from a $\mathscr{G}$-orbit describes a rational map in terms of "poles and residues."

The result, and its proof, constitute another example of the remarkable link between solutions to the anti-self-duality equations and holomorphic objects. (Both monopoles and Nahm's equations are examples of the anti-self-duality condition on $\mathbb{R}^{4}$, reduced by translational symmetries.) The correspondence with holomorphic objects functions essentially by forgetting part of the structure of a solution to the anti-self-duality equations. Conversely, one shows that one can recover the solution to the anti-self-duality equations from the holomorphic object, essentially by solving a variational problem. Other examples of this general pattern can be found in [D2, D3, UY, B].

The paper is organized as follows: the first four sections deal with the case of $S U(N)$; the fifth will show how to extend the result to the case of $S O(N)$ and $S p(N)$. In Sect. 1, following Donaldson, we will show how Nahm's equations divide into two parts, one invariant under a real group of gauge transformations, the other under a large complex group $\mathscr{G}$ of gauge transformations. In Sect. 2, we show how each $\mathscr{G}$-orbit contains an essentially unique solution to the real equations. Section 3 classifies solutions to the complex equations in terms of rational maps. Section 4 will interpret this rational map in terms of the twistor construction of monopoles, generalizing $[\mathrm{Hu}]$.

\section{Nahm's Equations and Nahm Complexes}

We begin, as in [D1], by setting $B(z)=\sum_{i=1}^{3}{ }^{i} T(z) d p_{i}$. (We drop the subscript index where we are not concerned about which interval $\left(\mu_{j}, \mu_{j+1}\right)$ we are discussing, or when this is implicitly obvious.) Nahm's equations are then equivalent to the anti-self-duality equations $d B+B \wedge B=-*(d B+B \wedge B)$ on the 4-space $\mathbb{R}^{4}=\left\{\left(z, p_{1}, p_{2}, p_{3}\right)\right\}$ : to get Nahm's equations, one has simply gauged the $d z$ component of $B$ to zero. It is then natural to reinsert this (skew-adjoint) component ${ }^{0} T(z)$ into the equations; setting

$$
\nabla_{z}\left({ }^{i} T\right)=\frac{d^{i} T}{d z}+\left[{ }^{0} T,{ }^{i} T\right]
$$

Nahm's equations become 


$$
\nabla_{z}\left({ }^{i} T\right)+\frac{1}{2} \sum_{j, k=1}^{3} \varepsilon_{i j k}\left[{ }^{j} T,{ }^{k} T\right]=0, \quad i=1,2,3 .
$$

This equation is invariant under unitary gauge transformations; if $u(z)$ is such a transformation, then

$$
\begin{aligned}
& u\left({ }^{i} T\right)=u^{i} T u^{-1}, \quad i=1,2,3 \\
& u\left({ }^{0} T\right)=u^{0} T u^{-1}-\frac{d u}{d z} u^{-1}
\end{aligned}
$$

maps one solution of (1.2) to another.

The isomorphisms $\mathbb{R}^{3}=\mathbb{C} \times \mathbb{R}$ in the hypotheses of the theorem allow one to introduce complex coordinates $\left(z+i p_{1}\right),\left(p_{2}+i p_{3}\right)$ and to write, in a corresponding fashion

$$
\alpha=\frac{1}{2}\left({ }^{0} T+i^{1} T\right), \quad \beta=\frac{1}{2}\left({ }^{2} T+i^{3} T\right) .
$$

Nahm's equations then become:

1. The "complex equation",

$$
\frac{d \beta}{d z}+2[\alpha, \beta]=0
$$

2. The "real equation",

$$
F(\alpha, \beta)=\frac{d}{d z}\left(\alpha+\alpha^{*}\right)+2\left(\left[\alpha, \alpha^{*}\right]+\left[\beta, \beta^{*}\right]\right)=0
$$

Let $g(z)$ lie in $G l(m, \mathbb{C})$ : setting

$$
g(\alpha)=g \alpha g^{-1}-\frac{1}{2} \frac{d g}{d z} g^{-1}, \quad g(\beta)=g \beta g^{-1},
$$

one finds that such a transformation preserves solutions to the complex equation; the real equation, on the other hand, is preserved only if $g(z)$ is unitary.

We now consider boundary behaviour, on an interval $\left(\mu_{j}, \mu_{j+1}\right)$.

Definition. $\mu_{j}$ is a superior (respectively inferior, neutral) boundary point of $\left(\mu_{j}, \mu_{j+1}\right)$ if $m_{j}>m_{j-1}$ (respectively $<$, $=$ ).

$\mu_{j+1}$ is a superior (respectively inferior, neutral) boundary point of $\left(\mu_{j}, \mu_{j+1}\right)$ if $m_{j}>m_{j+1}$ (respectively $<,=$ ).

At a boundary point $\mu_{j}$, we will denote by

$-k$ or $k_{j}$ the absolute value $\left|m_{j}-m_{j-1}\right|$ of the "jump"

- $\bar{m}$ or $\bar{m}_{j}$ the maximum of $\left(m_{j}, m_{j-1}\right)$

- $\underline{m}$ or $\underline{m}_{j}$ the minimum of $\left(m_{j}, m_{j-1}\right)$

$-\underline{j}$ the index of the smaller dimension (either $(j-1)$ or $j$ ).

At a boundary point $\mu$ of $\left(\mu_{j}, \mu_{j+1}\right)$, setting $t=z-\mu$, the boundary conditions of a solution to Nahm's equations are then, up to a unitary conjugation:

If $\mu$ is inferior, $\alpha_{j}, \beta_{j}$ are analytic at $t=0$, with values $\underline{\alpha}, \underline{\beta}$ at $t=0$.

If $\mu$ is neutral, $\alpha_{j}, \beta_{j}$ are analytic at $t=0$. 
If $\mu$ is superior, splitting $\mathbb{C}^{m_{j}}$ as $\mathbb{C}^{m} \oplus \mathbb{C}^{k}$, one has, near $t=0$ :

$$
\alpha_{j}=\left(\begin{array}{cc}
U & t^{(k-1) / 2} V \\
t^{(k-1) / 2} W & X
\end{array}\right), \quad \beta_{j}=\left(\begin{array}{cc}
P & t^{(k-1) / 2} Q \\
t^{(k-1) / 2} R & S
\end{array}\right) .
$$

with: i) $U, V, W$ and $P, Q, R$ analytic at $t=0$.

ii) $X, S$ are meromorphic, with simple poles at $t=0$, and residues $x, s$, such that, in an appropriate basis:

$$
\begin{gathered}
x=\operatorname{diag}\left(\frac{-(k-1)}{4}, \frac{2-(k-1)}{4}, \ldots, \frac{(k-1)}{4}\right), \\
s=\left[\begin{array}{ccccc}
0 & \cdots \cdots \cdots \cdots \cdots & 0 \\
1 & 0 & & \vdots \\
\vdots & 1 & \ddots & \vdots \\
\vdots & \ldots \ldots \cdots 1 & \vdots
\end{array}\right] .
\end{gathered}
$$

Furthermore, at $\mu_{j}, \alpha, \beta$ satisfy the patching conditions:

If $m_{j} \neq m_{j-1}\left(\mu_{j}\right.$ not neutral), then the limits $\underline{\alpha}, \underline{\beta}$ of (1.8a) are equal to the $U(0), P(0)$ of $(1.8 \mathrm{c})$.

If $m_{j}=m_{j-1}\left(\mu_{j}\right.$ neutral), one has limits $\alpha_{ \pm}, \beta_{ \pm}$from both sides of $\mu_{j}$; there then exist column vectors $u, w$ in $\mathbb{C}^{m_{j}}$ with

$$
\begin{gathered}
\beta_{+}-\beta_{-}=-\frac{1}{2} u w^{T}, \\
\left(\alpha_{+}+\alpha_{+}^{*}\right)-\left(\alpha_{-}+\alpha_{-}^{*}\right)=\frac{1}{2}\left(-u \bar{u}^{T}+\bar{w} w^{T}\right) .
\end{gathered}
$$

One can then choose for each jumping point $\mu_{j}, a v_{j}$,

If $m_{j} \neq m_{j-1}$, let $v_{j} \in \mathbb{C}^{\grave{m}}$ be a unit vector in the $-\left(\frac{k-1}{4}\right)$ eigenspace

of the residue $x$ of $(1.8 \mathrm{c})$. There is an $S^{1}$ of possible choices.

If $m_{j}=m_{j-1}$, let $v_{j}$ be the couple $(u, w)$ of $(1.9 \mathrm{~b})$; when $(u, w) \neq(0,0)$, there is again an $S^{1}$ of possible choices; when $(u, w)=(0,0)$, the solution is continuous at $\mu_{j}$ and corresponds to a $U(N-1)$ monopole embedded in $S U(N)$.

We will now define our complex gauge transformations, taking these boundary conditions into account.

Definition (1.11). Let $\mathscr{G}$ be the set of $(N-1)$-tuples $g=\left(g_{1}, \ldots, g_{N-1}\right)$, with $g_{j}$ a $C^{1}$-map,

$$
g_{j}:\left[\mu_{j}, \mu_{j+1}\right] \rightarrow G l\left(m_{j}, \mathbb{C}\right)
$$

smooth on the interior, such that

1. At a superior boundary point $\mu, g_{j}$ preserves the decomposition $\mathbb{C}^{m_{j}}=$ $\mathbb{C}^{m}+\mathbb{C}^{k}$, and $g_{j}$ has off diagonal blocks whose derivatives are $O\left(t^{(k-1) / 2}\right)$.

2. $g$ satisfies the following patching condition i) at a non-neutral boundary point $\mu$, let $\bar{g}$ denote the limit from the superior 
side, and $\underline{g}$ the limit from the inferior side; let $h$ denote the $\underline{m} \times \underline{m}$ upper diagonal block of $\bar{g}$; then $h=g$.

ii) at a neutral boundary point, the limits from both sides coincide.

We define $\mathscr{G}_{\mathbb{R}} \subset \mathscr{G}$ to be the set of those $g$ 's with $g$ unitary.

Definition (1.12). A Nahm complex is a triple

with

$$
(\alpha, \beta, v)=\left(\left(\alpha_{1}, \ldots, \alpha_{N-1}\right),\left(\beta_{1}, \ldots, \beta_{N-1}\right),\left(v_{1}, \ldots, v_{N}\right)\right)
$$

$$
\alpha_{j}, \beta_{j}:\left(\mu_{j}, \mu_{j+1}\right) \rightarrow g l\left(m_{j}, \mathbb{C}\right)
$$

and i) if $m_{j} \neq m_{j-1}, v_{j} \in \mathbb{C}^{\tilde{m}_{j}}$

ii) if $m_{j}=m_{j-1}, v_{j}=\left(u_{j}, w_{j}\right) \in \mathbb{C}^{m_{j}} \times \mathbb{C}^{m_{j}}$ satisfying the conditions:

1. $\left(d \beta_{j} / d z\right)+2\left[\alpha_{j}, \beta_{j}\right]=0$.

2. $\alpha_{j}, \beta_{j}$ smooth in $\left(\mu_{j}, \mu_{j+1}\right)$.

3. $\alpha, \beta$ satisfy the boundary conditions $(1.8 \mathrm{a}, \mathrm{b}, \mathrm{c})$ up to the action of an element of $\mathscr{G}$.

4. $\beta$ (but not necessarily $\alpha$ ) satisfies the patching condition in $(1.9 \mathrm{a}, \mathrm{b})$.

5. For $m_{j} \neq m_{j-1}$, the $v_{j}$ satisfy $(1.10 \mathrm{a})$, but are not necessarily unit vectors. For $m_{j}=m_{j-1}, v_{j}$ is a couple $(u, w)$ satisfying $\left(\beta_{+}-\beta_{-}\right)=-\frac{1}{2} u w^{T}$, as in $(1.9 \mathrm{~b})$.

Definition (1.13). Two Nahm complexes $(\alpha, \beta, v)\left(\alpha^{\prime}, \beta^{\prime}, v^{\prime}\right)$ are equivalent if there exists $g \in \mathscr{G}$ with

$$
\begin{aligned}
g(\alpha) & =\alpha^{\prime}, \\
g(\beta) & =\beta^{\prime}, \\
g_{\bar{m}_{j}}\left(\mu_{j}\right)\left(v_{j}\right) & =v_{j}^{\prime} \quad \forall_{j} .
\end{aligned}
$$

A real Nahm complex is a Nahm complex such that

1. $\alpha, \beta$ solve the real equations.

2. $\alpha, \beta$ satisfy the boundary conditions $(1.8 \mathrm{a}, \mathrm{b}, \mathrm{c})$ up to the action of an element of $\mathscr{G}_{\mathbb{R}}$.

3. $\left(\alpha+\alpha^{*}\right)$ satisfies the patching condition corresponding to the one for $\alpha$ in $(1.9 \mathrm{a}, \mathrm{b})$.

4. When $m_{j} \neq m_{j-1}$, the $v_{j}$ are unit vectors, and when $m_{j}=m_{j-1}$ the $v_{j}$ satisfy $(1.10 \mathrm{~b})$.

Proposition (1.14). There is a natural bijective correspondence between

1. Solutions to Nahm's equations, satisfying the conditions of Theorem 2, along with $v_{j}$ satisfying $(1.10 a, b)$, (with $v_{j}$ unit vectors for $\left.m_{j} \neq m_{j-1}\right)$; modulo the action of $\prod_{j=1}^{N-1} U\left(m_{j}\right)$.

2. Real Nahm complexes, modulo the action of $\mathscr{G}_{\mathbb{R}}$.

Proof. Given a solution to Nahm's equations, one can, by unitary gauge transformations which are non-constant only on a compact subset of the 
intervals $\left(\mu, \mu_{j+1}\right)$ arrange for the boundary and patching conditions to be satisfied. One then has a real Nahm complex. Conversely, by going to a flat $\left(T_{0}=0\right)$ gauge, one obtains a solution to Nahm's equations.

Proposition (1.15). (“Normal form”).

a) Away from a boundary point, or at an inferior or neutral boundary point, the $\alpha$ and $\beta$ of a Nahm complex are locally equivalent (i.e., by an element of $\mathscr{G}$ ) to

$$
\alpha(z)=0, \quad \beta(z)=\beta_{0}, \quad \text { a constant } .
$$

b) At a superior boundary point $\mu$, setting $t=z-\mu$, a Nahm complex is locally equivalent to, in the splitting $\mathbb{C}^{m}=\mathbb{C}^{m} \oplus \mathbb{C}^{k}$ :

$$
\begin{aligned}
& \alpha(t)=\frac{1}{t}\left[\begin{array}{c|c}
0 & 0 \\
\hline 0 & -\frac{(k-1)}{4} \ddots^{(k-1)} \\
& \frac{(k-1}{4}
\end{array}\right]
\end{aligned}
$$

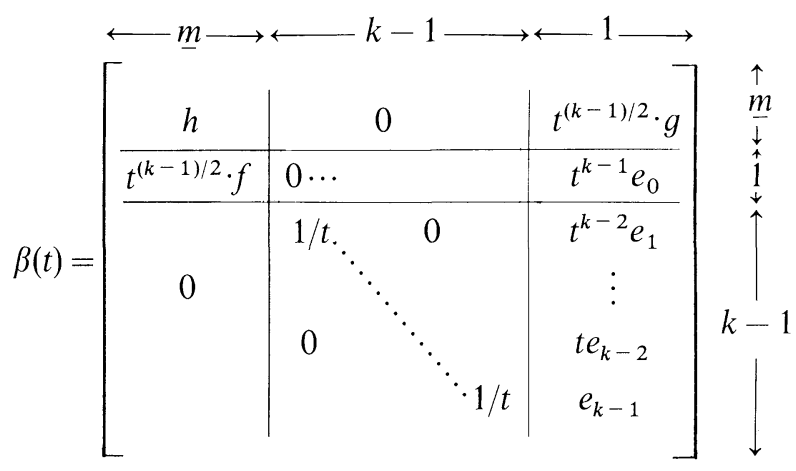

$$
\begin{aligned}
& v=\left(v_{i}\right), v_{i}=\delta_{i, \underline{m}+1}
\end{aligned}
$$

with $e_{i} \in \mathbb{C}, f: \mathbb{C}^{m} \rightarrow \mathbb{C}, g: \mathbb{C} \rightarrow \mathbb{C}^{m}, h: \mathbb{C}^{m} \rightarrow \mathbb{C}^{m}$ linear maps. Furthermore, $h$ is the limit of $\beta$ from the inferior side.

Proof. The complex equations, modulo gauge, are locally trivial; solving

$$
\frac{d w}{d z}=-2 \alpha w
$$

for a basis of vectors $w_{i}$, then performing the gauge transformation $S$ with $S^{-1}=\left(w_{1}, \ldots, w_{m_{j}}\right)$ gives the result in a). For b), one considers the equation for the transformed $w^{\prime}=D \cdot w$, where $D=\operatorname{diag}\left(1, \ldots, 1, t^{(k-1) / 2}, \ldots, t^{(k-1) / 2}\right)$. Using the boundary condition ( $1.8 \mathrm{a}$ ), the equation for $w^{\prime}$ has a regular singular point at $t=0$. Applying the theory of such o.d.e.'s (see, e.g. [Ha]), and transforming back to $w$, one sees that, as in [D1], 
1. There is a unique $w_{1}(t)$ satisfying (1.16) with

$$
\lim _{t \rightarrow 0}\left(t^{-(k-1) / 2} w_{1}(z)-v\right)=0 .
$$

2. Setting $w_{i}(t)=\beta^{i-1}(t) w_{1}(t)$, then $w_{i}(t)$ solves (1.16) and

$$
\lim _{t \rightarrow 0}\left(t^{(i-1)-(k-1) / 2} w_{i}(t)-x^{(i-1)} v\right)=0 .
$$

3. There are solutions $u_{1}(t) \cdots u_{m}(t)$ to (1.16), whose last $k$ components vanish at $t=0$ to order $(k+1) / 2$, and which are linearly independent at $t=0$.

Setting $\tilde{S}^{-1}(t)=\left(u_{1}(t), \ldots, u_{m}(t), w_{1}(t), \ldots, w_{k}(t)\right), \tilde{S}$ transforms one to a gauge with $\alpha=0$, and $\beta$ a constant matrix of the form

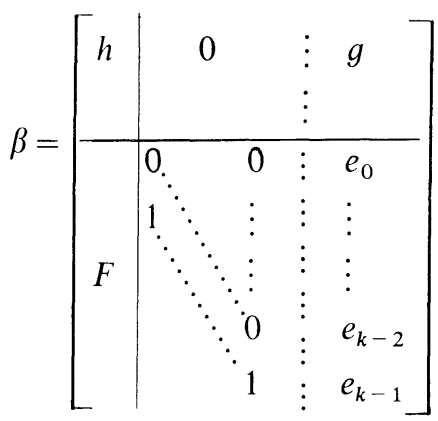

However $\tilde{S}$ is not regular at $t=0$; if one sets $S^{-1}=\tilde{S}^{-1} \cdot \operatorname{diag}\left(1 \cdots 1, t^{-(k-1) / 2}\right.$, $\left.t^{(-k+3) / 2}, \ldots, t^{(k-1) / 2}\right)$, then $S$ is regular at $t=0$, and, in fact, lies in $\mathscr{G}$. Under the action of $S, \alpha, \beta$ become of the form stated in the proposition; as $S$ lies in $\mathscr{G}$, one sees that all but the top row of $F$ is zero.

\section{Solving the Real Equation}

We now want to show that each $\mathscr{G}$-equivalence class of Nahm complexes contains a solution to the real equation, unique up to the action on $\mathscr{G}_{\mathbb{R}}$; gauging ${ }^{0} T$ to zero, this, by Proposition (1.14) is the same as showing that each equivalence class of Nahm complexes yields a solution to Nahm's equations, satisfying the conditions of Theorem 2; this solution is unique up to the action of the $U\left(m_{j}\right)$.

We prove this in two steps: we first show that we can obtain on each interval $\left(\mu_{j}, \mu_{j+1}\right)$ solutions of the desired form, satisfying the boundary conditions. We then show that the solutions on the different intervals can be patched together uniquely, to give us our global solution. The first step follows Donaldson [D1, Sect. 2] closely, so many details will be omitted.

A) Solution on an Interval. Fixing a solution $(\alpha, \beta)$ to the complex equations over an interval $[c, d]$, the real equation $F(g(\alpha, \beta))=0$ for $g$ a (sufficiently smooth) map

$$
g:[c, d] \rightarrow G l(m, \mathbb{C})
$$

has a nice variational description in terms of a functional $\mathscr{L}$ on the space of such 
maps. $F=0$ is the Euler-Lagrange equation for

$$
\mathscr{L}(g)=\int_{c}^{d}\left|g(\alpha)+g(\alpha)^{*}\right|^{2}+2|g(\beta)|^{2} d z .
$$

One notes that $\mathscr{L}$ is invariant under unitary gauge transformations. It is convenient to factor this out. Let $\mathscr{H}=G l(m, \mathbb{C}) / U(m)$ be the space of positive hermitian matrices; one defines

$$
h=h(g)=g^{*} g:(c, d) \rightarrow \mathscr{H} .
$$

Now suppose that $(\alpha, \beta)$ is such that it can be gauged to a constant $\left(0, \beta_{0}\right)$ on $[c, d]$. One has the existence and unicity property:

Proposition (2.3) [D1, pp. 395-397]. For any $h_{+}, h_{-}$in $\mathscr{H}$, there is a continuous $g:[c, d] \rightarrow G l(m, \mathbb{C})$, with $h(g)=h_{-}, h_{+}$at $c, d$ respectively such that $(\tilde{\alpha}, \widetilde{\beta})=g\left(0, \beta_{0}\right)$ satisfies the real equation $F(\tilde{\alpha}, \widetilde{\beta})=0$ in $(c, d)$.

If $g_{1}, g_{2}$ are any two such $g$ 's with $h_{1}(z)=g_{1}^{*} g_{1}, h_{2}(z)=g_{2}^{*} g_{2}$ taking on equal values at the endpoints $c, d$, then $h_{1}(z)=h_{2}(z)$ throughout $(c, d)$; the solution is thus unique up to a unitary gauge transformation.

The main tool for proving uniqueness is a "convexity lemma" for the eigenvalues of $h$ : for $h \in \mathscr{H}$ with eigenvalues $\lambda_{i}$, set $\Phi(h)=\log \max \left(\lambda_{i}\right)_{i=1}^{m} \in \mathbb{R}$.

Lemma (2.4) [D1, p. 396]. If $(\tilde{\alpha}, \tilde{\beta})=g(\alpha, \beta)$ over $[c, d]$, then

$$
\begin{gathered}
\frac{d^{2}}{d z^{2}} \Phi(h) \geqq-2(|F(\alpha, \beta)|+|F(\tilde{\alpha}, \widetilde{\beta})|), \\
\frac{d^{2}}{d z^{2}} \Phi\left(h^{-1}\right) \geqq-2(|F(\alpha, \beta)|+|F(\tilde{\alpha}, \widetilde{\beta})|)
\end{gathered}
$$

in the weak sense.

To extend (2.3) to an interval where $(\alpha, \beta)$ have poles, one must first put $(\alpha, \beta)$ into a "nice" form.

Lemma (2.5) [D1, p. 398]. Let $(\alpha, \beta, v)$ be a Nahm complex. Then there is an equivalent "nice" Nahm complex $\left(\alpha^{\prime}, \beta^{\prime}, v^{\prime}\right)$ with:

i) $F\left(\alpha^{\prime}, \beta^{\prime}\right)$ bounded,

ii) $\left|\alpha^{\prime}-\alpha^{\prime *}\right|$ bounded,

iii) $\left|v_{j}\right|=1$, when $m_{j} \neq m_{j-1}$.

iv) The residues of the matrices ${ }^{i} T$ corresponding to $\alpha^{\prime}, \beta^{\prime}$ at superior boundary points are conjugate under a $U(k)$ transformation to the standard irreducible representation $\tau_{i}$ of $S U(2)$ on $\mathbb{C}^{k} \subset \mathbb{C}^{\bar{m}}$.

With this, one has

Theorem (2.6) [D1, pp. 399-402]. Let $(\alpha, \beta, v)$ be a Nahm complex, nice in the sense of (2.5). Then there is a $g:\left[\mu_{j}, \mu_{j+1}\right] \rightarrow G l\left(m_{j}, \mathbb{C}\right)$ continuous, smooth on the interior, with $h\left(\mu_{j}\right)=h\left(\mu_{j+1}\right)=1$, and $(d g / d z)$ bounded in $\left(\mu_{j}, \mu_{j+1}\right)$ such that

1. $g(\alpha, \beta, v)$ solves the real equation, with $\alpha=\alpha^{*}$ over $\left(\mu_{j}, \mu_{j+1}\right)$, i.e., defines $a$ solution to Nahm's equations. 
2. This solution is bounded, continuous at an inferior or neutral boundary point; at a superior boundary point $\mu$ there is a unitary matrix $v$ with

$$
\left|\left(v^{i} T v^{-1}-\left(\tau_{i} /(z-\mu)\right)\right)\right|
$$

bounded.

Any two such g's give solutions which are conjugate by a unitary matrix.

There remain two properties of the solutions ${ }^{i} T(z)$ on $\left(\mu_{j}, \mu_{j+1}\right)$ to be proven. One is that the ${ }^{i} T$ have the correct analytic and meromorphic behaviour; the other is that they have the correct block decomposition at a superior boundary point.

In the $S U(2)$ case treated in [D1], to show that the solutions are meromorphic, one appeals to the theorem of Hitchin [Hi], which says that the ${ }^{i} T$ of $(2.6)$ yield an $S U(2)$ monopole; this in turn gives one enough regularity to assert that the ${ }^{i} T$ have the appropriate analyticity. In the $S U(N)$ case, one cannot appeal to the similar theorem in $[\mathrm{HuM}]$; the reason is that the construction of the monopole from the ${ }^{i} T$ in this case assumes the analytic behaviour of the ${ }^{i} T$. One must therefore proceed differently.

We will exploit the fact that Nahm's equations can be expressed in terms of flows on the Jacobian of a curve embedded in $T \mathbb{P}_{1}$. We briefly recall the pertinent details of this construction; more can be found in [HuM], [Hi]. Let $\zeta$ be a standard coordinate of $\mathbb{P}_{1}$, and let $\eta \rightarrow \eta(d / d \zeta)$ be the associated fiber coordinate in $T \mathbb{P}_{1}$. Now write

$$
\begin{aligned}
A(z, \zeta) & =A_{0}(z)+A_{1}(z) \zeta+A_{2}(z) \zeta^{2} \stackrel{\text { def }}{=}\left({ }^{2} T+i^{3} T\right)(z)+2 i^{1} T(z) \zeta+\left({ }^{2} T-i^{3} T\right)(z) \zeta^{2} \\
& =2 \beta(z)+4 i \alpha(z) \zeta-2 \beta^{*}(z) \zeta^{2} .
\end{aligned}
$$

Nahm's equations can be rewritten as:

$$
\frac{d A}{d z}+\left[\frac{A_{1}}{2}+A_{2} \zeta, A\right]=0 .
$$

Let $\mathcal{O}(k)$ denote the lift to $T \mathbb{P}_{1}$ of the standard line bundles $\mathcal{O}(k)$ on $\mathbb{P}_{1}$; one also defines a line bundle $L^{z}$, for $z \in \mathbb{C}$ by the transition function $\exp (z \eta / \zeta)$ from $\{\zeta \neq \infty\}$ to $\{\zeta \neq 0\}$. Now define the sheaf $X_{z}$ over $T \mathbb{P}_{1}$ :

$$
0 \rightarrow \mathcal{O}(-2)^{\oplus m} \stackrel{(\eta \mathrm{Id}-A(z, \zeta))}{\longrightarrow} \mathcal{O}^{\oplus m} \rightarrow X_{z} \rightarrow 0 .
$$

$X_{z}$ is supported on the (compact) curve

$$
S=\{(\eta, \zeta) \mid \operatorname{det}(\eta \mathrm{Id}-A(z, \zeta))=0\}
$$

Because Nahm's equations are in Lax form, $S$ is an invariant of the flow. One can show that if $A$ solves Nahm's equations, $X_{z}=X_{0} \otimes L^{z}$. Also, one can prove that

$$
H^{0}\left(S, X_{z}\right) \cong H^{0}\left(S \cap\left(\zeta=\zeta_{0}\right), X_{z}\right) \text { for all } \zeta_{0} .
$$

To obtain $A(z, \zeta)$ from a flow $X_{0} \otimes L^{z}$, one

1. forms the (rank $m$ ) vector bundle $V$ over $\mathbb{C}$ whose fiber at a generic $z$ will be $H^{0}\left(S, X_{z}\right)$ (more properly, $V$ will be a direct image sheaf under a projection $S \times \mathbb{C} \rightarrow \mathbb{C})$. 
2. One then defines a natural geometric endomorphism $\tilde{A}(z, \zeta)$ of $V_{z}$, for the $z$ verifying (2.10), by the diagram:

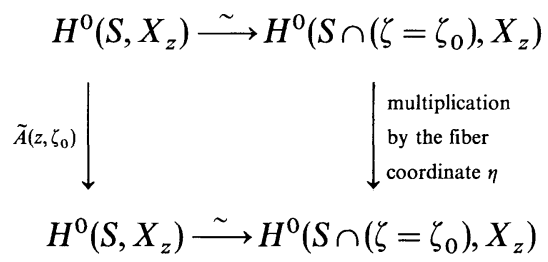

Note that as the sections on the left are supported on discrete points distinct from $\eta=\infty$, the map is well defined.

3. One trivializes $V$ by an appropriate connection; $\tilde{A}(z, \zeta)$ then becomes a matrix $A(z, \zeta)$, and solves Nahm's equations. One such connection could just be the connection $\nabla_{0}$ defined by evaluation at $\zeta=0$ (in an appropriate trivialization). In a $\nabla_{0}$ constant basis, by $(2.11)$, one has that $\tilde{A}(z, 0)=2 \beta(z)$ is constant in $z$. This, however, is incorrect; the connection appropriate to solving Nahm's equations is $\nabla=\nabla_{0}+\left(A_{1}(z) / 2\right) d z$.

It is clear from the above that the solution one obtains is analytic, whenever (2.10) holds, and so wherever $A(z, \zeta)$ is finite. There remains the problem of a superior boundary point. Note that steps 1 and 2 are essentially algebraic; $\tilde{A}(z, \zeta)$ is meromophic in any geometrically defined basis of $V$. In particular, with respect to the $\nabla_{0}$-flat basis, the matrix of $\tilde{A}$ is meromorphic. We will denote this matrix by $B(z, \zeta)$. Any essential singularity of the solution $A(z, \zeta)$ of Nahm's equations is then due to the passage from a $\nabla_{0}$-flat basis to a $\nabla$-flat basis. Let $f(z)$ be the matrix linking the two; one has:

$$
\begin{gathered}
A(z, \zeta)=f(z) B(z, \zeta) f(z)^{-1}, \\
A_{1}(z)=-2 \frac{d f}{d z} f^{-1} \\
B_{1}(z)=-2 f^{-1} \frac{d f}{d z}
\end{gathered}
$$

Now suppose one is at a superior boundary point of $\left(\mu_{j}, \mu_{j+1}\right)$; for definiteness, take this to be $\mu_{j}$. One can choose the matrix $g\left(\mu_{j}\right)$ of Theorem (2.6) to preserve the decomposition $\mathbb{C}^{m_{j}}=\mathbb{C}^{m_{J}-1} \oplus \mathbb{C}^{k}$ at $\mu_{j}$. Furthermore, referring to Proposition (1.15) and the fact that $\beta=$ constant in the $\nabla_{0}$-gauge, to Lemma (2.5) and to Theorem (2.6), one sees that near $\mu_{j}$

$$
f=g \cdot k \cdot \operatorname{diag}\left(t^{\lambda_{2}}\right)
$$

with $t=\left(z-\mu_{j}\right),\left(\lambda_{1}, \ldots, \lambda_{m_{j}}\right)=(0 \ldots 0,(k-1) / 2,(k-3) / 2, \ldots,-(k-1) / 2), k \in \mathscr{G}$, and $d g / d t$ bounded along the real axis at $\mu_{j}$. Differentiating, one obtains:

$$
(g k)^{-1} \frac{d(g k)}{d z}=\operatorname{diag}\left(t^{\lambda_{i}}\right)\left(-\frac{B_{1}}{2}-\operatorname{diag}\left(\frac{\lambda_{i}}{t}\right)\right) \operatorname{diag}\left(t^{-\lambda_{i}}\right) .
$$

As $B_{1}$ is meromorphic, one sees that $M=(g k)^{-1}(d(g k) / d z)$ has the block decom- 
position near $\mu_{j}$

$$
M=\left(\begin{array}{lr}
P(t) & t^{\rho / 2} Q(t) \\
t^{\rho / 2} R(t) & S(t)
\end{array}\right)
$$

with $P, Q, R, S$ meromorphic, and $\rho=0,1$ with $\rho,(k-1)$ having the same parity. As $d(g k) / d z$ is bounded, $P, Q, R, S$ are in fact analytic at $t=0$. Solving for $(g k)$, one sees that $(g k)$ has a similar decomposition, with $Q, R$ vanishing at $t=0$. In turn, the ${ }^{i} T$ 's of (2.6) also have a similar decomposition, with $S$ meromorphic, $P, Q, R$ analytic, and for $k>1, t^{\rho / 2} Q, t^{\rho / 2} R$ vanishing at $t=0$.

To complete our discussion of the solutions on the interval, one must show that the off-diagonal blocks vanish to the correct order at $t=0$, for a superior boundary point with $k>1$. One notes that, setting the off diagonal blocks in the Nahm complex to zero, and applying (2.6), one obtains a different solution ${ }^{i} T^{\prime}$ which is block diagonal, i.e., a sum of a $g l(k)$ and a $g l(\underline{m})$ solution. We view ${ }^{i} T$ as a perturbation of ${ }^{i} T^{\prime}$, and set

$$
\Delta^{i} T={ }^{i} T-{ }^{i} T^{\prime}=\left(\begin{array}{ll}
a_{i} & b_{i} \\
c_{i} & d_{i}
\end{array}\right)
$$

From what we know from the above, $a_{i}, b_{i}, c_{i}, d_{i}$ are Frobenius series $t^{\gamma} \times$ (analytic in $t$ ), with $\gamma \geqq 0$ for $d_{i}, \gamma>0$ for $b_{i}, c_{i}, a_{i}$. One has the equation for $\Delta^{i} T$ :

$$
\frac{d\left(\Delta^{i} T\right)}{d t}+\frac{1}{2} \Sigma \varepsilon_{i j k}\left(\left[\Delta^{j} T,{ }^{k} T^{\prime}\right]+\left[{ }^{j} T, \Delta^{k} T\right]+\left[\Delta^{j} T, \Delta^{k} T\right]\right)=0 .
$$

This equation has a linear term with a simple pole at $t=0$, and a smooth quadratic term; as we know a priori that the solutions are $t^{\gamma}$ (analytic in $t$ ) $\gamma \geqq 0$, we find that, as in the linear case, $-\gamma$ must be an eigenvalue of the residue of the linear term. As

$$
\operatorname{res}\left({ }^{i} T^{\prime}\right)=\left(\begin{array}{ll}
0 & 0 \\
0 & \tau_{i}
\end{array}\right)
$$

with $\tau_{i}(k \times k)$ matrices defining an irreducible representation of $s u(2)$ one finds, for the terms $b_{i}$, that

$$
\frac{d}{d z}\left(\begin{array}{l}
b_{1} \\
b_{2} \\
b_{3}
\end{array}\right)+\frac{1}{t}\left(\begin{array}{ccc}
0 & -\tau_{3} & \tau_{2} \\
\tau_{3} & 0 & -\tau_{1} \\
-\tau_{2} & \tau_{1} & 0
\end{array}\right)\left(\begin{array}{l}
b_{1} \\
b_{2} \\
b_{3}
\end{array}\right)+\text { lower order }=0
$$

and a similar equation for the $c_{i}$. This polar term has a representation theoretic interpretation; each column of the matrix of $b$ 's can be thought of as an element of the representation $S^{2} \otimes S^{k-1}$ of $s u(2)$; in these terms, the action of the matrix of $\tau$ 's on the $b$ 's is given by $\Sigma e_{i} \otimes e_{i}$, where the $e_{i}$ 's form the standard basis of $s u(2)$. In terms of Casimir operators:

$$
\Sigma e_{i} \otimes e_{i}=\frac{1}{2}\left(C\left(S^{2} \otimes S^{k-1}\right)-C\left(S^{2}\right) \otimes 1-1 \otimes C\left(S^{k-1}\right)\right) .
$$

Decomposing $S^{2} \otimes S^{k-1}$ into irreducibles as $S^{k+1} \oplus S^{k-1} \oplus S^{k-3}$, and using $C\left(S^{k}\right)=-k(k+2) / 4$, one gets as possibilities for $\gamma:(k-1) / 2$ on $S^{k+1},-1$ on $S^{k-1}$ 
$-(k+1) / 2$, on $S^{k-3}$. The only positive $\gamma$ is $(k-1) / 2$, and so $b_{i}$ vanishes to order $(k-1) / 2$. The proof for the $c_{i}$ is the same.

Remark: Once one knows the boundary behaviour of the ${ }^{i} T$, it is easy to show, using (2.13), that if $g\left(\mu_{j}\right)$ respects the decomposition $\mathbb{C}^{m_{j}}=\mathbb{C}^{\underline{m}} \oplus \mathbb{C}^{k}$ at a superior boundary point, then $g$ satisfies at $\mu_{j}$ the constraint for lying in $\mathscr{G}$; in fact, the $g$ of (2.15) has analytic diagonal blocks, and off-diagonal blocks of the form $t^{(k+1) / 2} \cdot$ analytic.

Given a Nahm complex, then, we have produced a solution to Nahm's equations over $\left(\mu_{j}, \mu_{j+1}\right)$ with the correct boundary behaviour. Alternately, we have, in the orbit of $\mathscr{G}$, a solution to the real equations.

There arises the question of how many different solutions one can produce in this way. Note that one obtains gauge equivalent solutions from $g, g^{\prime}$ if $g^{\prime} g^{-1}\left(\mu_{j}\right)$, $g^{\prime} g^{-1}\left(\mu_{j+1}\right)$ lie in $U\left(m_{j}\right)$. At a neutral or inferior boundary point $\mu$ one's degree of freedom is thus $g^{*} g(\mu) \in \mathscr{H}\left(m_{j}\right)=G l\left(m_{j}, \mathbb{C}\right) / U\left(m_{j}\right)$. At a superior boundary point, one has "used up" part of this freedom to produce the "nice" Nahm complex of (2.5); $g$ lives in $G l(m) \times U(k)$ and so the degrees of freedom, modulo unitary transformations, is $\overline{\mathscr{H}}(\underline{m})$. Summing this up:

Theorem (2.18). Let $r_{j}=\min \left(m_{j}, m_{j-1}\right)$. Let $(\alpha, \beta, v)$ be a Nahm complex. The solutions to the real equation, over the interval $\left(\mu_{j}, \mu_{j+1}\right)$ lying in the $\mathscr{G}$-orbit of $(\alpha, \beta, v)$ are, modulo the action of $\mathscr{G}_{\mathbb{R}}$, parametrized by

$$
\mathscr{H}\left(r_{j}\right) \times \mathscr{H}\left(r_{j+1}\right)
$$

with the convention $\mathscr{H}(0)=\{$ point $\}$.

B) Patching Together the Intervals. We now show inductively in $(j-i)$ that for a given Nahm complex the set $m_{i j}$ of solutions on an interval $\left(\mu_{i}, \mu_{j}\right), i<j$ satisfying the boundary conditions at all boundary points and the patching conditions at the "interior" boundary points $\mu_{k}, i<k<j$, modulo $\mathscr{G}_{\mathbb{R}}$, is parametrized by $\mathscr{H}\left(r_{i}\right) \times \mathscr{H}\left(r_{j}\right)$. As $\mathscr{H}\left(r_{1}\right) \times \mathscr{H}\left(r_{N}\right)$ is a singleton, this will prove existence and unicity.

Consider then $\left(\mu_{i}, \mu_{j}\right),\left(\mu_{j}, \mu_{k}\right), i<j<k$. We would like to show that fixing $h_{i} \in \mathscr{H}\left(r_{i}\right), h_{k} \in \mathscr{H}\left(r_{k}\right)$ there is a unique $h_{j} \in \mathscr{H}\left(r_{j}\right)$ such that the solutions on $\left(\mu_{i}, \mu_{j}\right),\left(\mu_{j}, \mu_{k}\right)$, corresponding to $\left(h_{i}, h_{j}\right),\left(h_{j}, h_{k}\right)$, respectively satisfy the patching condition at $\mu_{j}$.

Without loss of generality, let us take $h_{i}=h_{k}=1$. Let us fix a "base" solution $(\alpha, \beta)$ for both intervals corresponding to $h_{j}=1$. For each $h_{j}$, there is a $g \in \mathscr{G}$ such that $h=g^{*} g$ satisfies $h\left(\mu_{i}\right)=1, h\left(\mu_{j}\right)=h_{j}, h\left(\mu_{k}\right)=1$, and the transform $(\tilde{\alpha}, \widetilde{\beta})=g(\alpha, \beta)$ also provides a solution over $\left(\mu_{i}, \mu_{j}\right)$ and $\left(\mu_{j}, \mu_{k}\right)$. The key ingredient is:

Lemma (2.19). Let $h$ be as above, and define $\varphi=\Phi(h), \psi=\Phi\left(h^{-1}\right)$ as in (2.4). Let $\Delta \varphi^{\prime}, \Delta \psi^{\prime}$, denote the jumps in $\varphi^{\prime}, \psi^{\prime}$ at $\mu_{j}$. (Here ' denotes $d / d z$.) Then, for some constant $K$,

$$
\begin{aligned}
& \Delta \varphi^{\prime} \leqq-\varphi\left(\mu_{j}\right)\left(\frac{1}{\left(\mu_{k}-\mu_{j}\right)}+\frac{1}{\left(\mu_{j}-\mu_{i}\right)}\right)+K, \\
& \Delta \psi^{\prime} \leqq-\psi\left(\mu_{j}\right)\left(\frac{1}{\left(\mu_{k}-\mu_{j}\right)}+\frac{1}{\left(\mu_{j}-\mu_{i}\right)}\right)+K .
\end{aligned}
$$


Proof. One begins by noting that, as $h$ is analytic near any $\mu_{l}$, one sided limits $\varphi_{ \pm}^{\prime}\left(\mu_{l}\right)$ of $\varphi^{\prime}$ exist at $\mu_{l}$. Also, at any interior boundary point $\mu_{l}$ of $\left(\mu_{i}, \mu_{j}\right)$, from the equation

$$
\frac{1}{2} g^{\prime}=g \alpha-\tilde{\alpha} g,
$$

and the fact that both $\alpha, \tilde{\alpha}$ satisfy the patching conditions, one gets a similar patching for $g^{\prime}$; this continuity of $g^{\prime}$ gives us

$$
\varphi_{-}^{\prime}\left(\mu_{l}\right) \leqq \varphi_{+}^{\prime}\left(\mu_{l}\right)+k_{1} .
$$

From the fact (2.4) that $\varphi^{\prime \prime} \geqq 0$ weakly, one easily obtains, by Rolle's theorem, that on each interval $\left(\mu_{l}, \mu_{l+1}\right), i \leqq l<l+1 \leqq j$,

$$
\varphi_{+}^{\prime}\left(\mu_{l}\right)\left(\mu_{l+1}-\mu_{l}\right) \leqq \varphi\left(\mu_{l+1}\right)-\varphi\left(\mu_{l}\right) \leqq \varphi_{-}^{\prime}\left(\mu_{l+1}\right)\left(\mu_{l+1}-\mu_{l}\right),
$$

and so

$$
\varphi\left(\mu_{l+1}\right)-\varphi\left(\mu_{l}\right) \leqq \varphi_{-}^{\prime}\left(\mu_{j}\right)\left(\mu_{l+1}-\mu_{l}\right)+k_{2},
$$

and so after summing, $\left(\varphi\left(\mu_{i}\right)=\varphi\left(\mu_{k}\right)=0\right)$,

Similarly,

$$
\varphi\left(\mu_{j}\right) \leqq \varphi_{-}^{\prime}\left(\mu_{j}\right)\left(\mu_{j}-\mu_{i}\right)+k_{3} .
$$

$$
\left(\mu_{k}-\mu_{i}\right) \varphi_{+}^{\prime}\left(\mu_{j}\right) \leqq-\varphi\left(\mu_{j}\right)+k_{4} .
$$

which is what we need. The proof for $\psi$ is similar.

Proposition (2.20). Let $\mu_{j}$ be a non-neutral boundary point. Fixing $h_{i}, h_{k}$, there is a unique $h_{j} \in \mathscr{H}\left(r_{j}\right)$ such that the corresponding solutions on $\left(\mu_{i}, \mu_{j}\right)$ and $\left(\mu_{j}, \mu_{k}\right)$ satisfy the patching conditions at $\mu_{j}$.

Proof. We compute the norm $\operatorname{tr}\left(\left(\Delta\left(\tilde{\alpha}+\tilde{\alpha}^{*}\right)\right)^{2}\right)$ of the jump $\Delta\left(\tilde{\alpha}+\tilde{\alpha}^{*}\right)$ at $\mu_{j}$ as a function of $\Delta \alpha, \Delta \alpha^{*}$, and $h$, and find:

$$
\operatorname{tr}\left(\Delta\left(\tilde{\alpha}+\tilde{\alpha}^{*}\right)^{2}\right)=\operatorname{tr}\left[h(\Delta \alpha) h^{-1}+\Delta \alpha^{*}-\frac{1}{2}\left(\Delta h^{\prime}\right) h^{-1}\right]^{2} .
$$

where $h, \Delta h^{\prime}$ are evaluated at $\mu_{i}$.

Let us suppose that $h\left(\mu_{j}\right)$ has distinct eigenvalues, and diagonalize $h$ by unitary gauge transformations on each side of $\mu_{j}$. This modifies $\Delta \alpha$ by an essentially irrelevant unitary factor. Setting $h=\operatorname{diag}\left(e^{t_{i}}\right)$ and expanding (2.21), one obtains

$$
\sum_{i j}\left(\Delta \alpha_{i j} \Delta \alpha_{j i}+\Delta \bar{\alpha}_{j i} \Delta \bar{\alpha}_{i j}\right)+\frac{1}{4} \sum_{i}\left(\Delta t_{i}^{\prime}\right)^{2}-\sum_{i}\left(\Delta \alpha_{i i}+\Delta \bar{\alpha}_{i i}\right) \Delta t_{i}^{\prime}+\sum_{i, j} e^{t_{i}-t_{j}} \Delta \alpha_{i j} \Delta \bar{\alpha}_{i j},
$$

where $t_{i}=t_{i}\left(\mu_{j}\right), \Delta t_{i}^{\prime}=\Delta t_{i}^{\prime}\left(\mu_{j}\right)$. This is of the form (quadratic polynomial in $\left.\Delta t_{i}^{\prime}\right)+$ positive. As $\Delta \varphi^{\prime}=\Delta\left(\max t_{i}\right)^{\prime}, \Delta \psi^{\prime}=\Delta\left(\min t_{i}\right)^{\prime}$, one obtains as the quadratic term in the polynomial is positive, a bound

$$
\operatorname{tr}\left(\Delta\left(\tilde{\alpha}+\tilde{\alpha}^{*}\right)^{2}\right) \geqq c\left(\left(\Delta \varphi^{\prime}\right)^{2}+\left(\Delta \psi^{\prime}\right)^{2}\right)+K
$$

for some positive $c$, which extends to the case of non-distinct eigenvalues. As $h_{j}$ tends to infinity in $\mathscr{H}\left(r_{j}\right)$, the same must be true of either $\varphi^{2}$ or $\psi^{2}$; by Lemma (2.19), one of $\Delta \varphi^{\prime}, \Delta \psi^{\prime}$ also tends to infinity.

In short, the map $\rho: \mathscr{H}\left(r_{j}\right) \rightarrow \mathbb{R}^{+}$, sending $h_{j}$ to $\operatorname{tr}\left(\Delta\left(\tilde{\alpha}+\tilde{\alpha}^{*}\right)^{2}\right)$, is proper, and so it has a minimum. 
There are two possibilities for a critical point $(\tilde{\alpha}, \tilde{\beta})$ of $\rho$ : either $\Delta\left(\tilde{\alpha}+\tilde{\alpha}^{*}\right)=0$, or the differential of $\Delta\left(\tilde{\alpha}+\tilde{\alpha}^{*}\right)$ is singular. We now exclude the latter. We choose a unitary gauge so that $\tilde{\alpha}=\tilde{\alpha}^{*}$ : performing an infinitesimal gauge change $(1+s x)$ preserving the real equations with $x$ self adjoint, $x\left(\mu_{i}\right)=x\left(\mu_{k}\right)=0$, we obtain

$$
\frac{d}{d s}\left(\Delta\left(\tilde{\alpha}+\tilde{\alpha}^{*}\right)\right)=-2\left(\Delta x^{\prime}+\Delta x^{*}\right)
$$

By the infinitesimal version of (2.19), the norm of $\Delta x^{\prime}$ is bounded below by that of $x\left(\mu_{j}\right)$ and so the differential is nowhere singular.

The only critical points of $\rho$ are the zeros of $\Delta\left(\tilde{\alpha}+\tilde{\alpha}^{*}\right)$; as the differential of $\Delta\left(\tilde{\alpha}+\tilde{\alpha}^{*}\right)$ is nowhere zero, the zeroes are isolated; a min-max argument shows that it is unique.

Proposition (2.21). Let $\mu_{j}$ be a neutral boundary point. Then, as above, fixing $h_{i}, h_{k}$ there is a unique $h_{j}$ such that the corresponding solutions on $\left(\mu_{i}, \mu_{j}\right),\left(\mu_{j}, \mu_{k}\right)$ satisfy the patching conditions $(1.9 b)$ at $\mu_{j}$.

Proof. Again, let $\alpha, \beta$ denote our "base" solution; by the proof of the preceding proposition, we can suppose $\Delta \alpha=0$. Let $u, w$ be the vectors such that $\Delta \beta=-\frac{1}{2} u w^{T}$. Let $g$ be a gauge transformation corresponding to $h_{j}:$ if $(\tilde{\alpha}, \widetilde{\beta})=g(\alpha, \beta)$, we want, at $\mu_{j}$

$$
\Delta\left(\tilde{\alpha}+\tilde{\alpha}^{*}\right)=\frac{1}{2}\left(-g u u^{*} g^{*}+g^{-1^{*}} \bar{w} w^{T} g^{-1}\right),
$$

and so, at $\mu_{j}$, we want

$$
M \stackrel{\text { def }}{=} \Delta\left(g^{\prime}\right) g^{-1}+g^{-1^{*}} \Delta\left(g^{\prime}\right)^{*}-g u u^{*} g^{*}+g^{-1^{*}} \bar{w} w^{T} g^{-1}=0 .
$$

Computing $\operatorname{tr}\left(M^{2}\right)=\operatorname{tr}\left(g^{*} M^{2} g^{*-1}\right)$, one finds:

$$
\operatorname{tr}\left(\Delta\left(h^{\prime}\right) h^{-1}-h u u^{*}+\bar{w} w^{T} h^{-1}\right)^{2} .
$$

Again, by a unitary change of gauge, it is sufficient to consider $h=\operatorname{diag}\left(e^{t_{i}}\right)$. The diagonal in $g^{*} M g^{*-1}$ contributes to $\operatorname{tr}\left(M^{2}\right)$ terms of the form:

$$
\rho_{i}^{2}=\left(\Delta\left(t_{i}^{\prime}\right)-e^{t_{i}} u_{i} \bar{u}_{i}+w_{i} \bar{w}_{i} e^{-t_{i}}\right)^{2}
$$

and the off-diagonal, terms of the form:

$$
\sigma_{i j}=e^{t_{i}+t_{j}} u_{i} \bar{u}_{i} u_{j} \bar{u}_{j}+e^{-t_{i}-t_{j}} w_{i} \bar{w}_{i} w_{j} \bar{w}_{j}-u_{i} \bar{u}_{j} w_{j} \bar{w}_{i}-u_{j} \bar{u}_{i} w_{i} \bar{w}_{j} .
$$

The $\sigma_{i j}$ 's are of the form (positive-constant): as for the $\rho_{i}$, ordering the eigenvalues by $t_{1} \leqq \cdots \leqq t_{m}$, one has that either $\rho_{1} \rightarrow+\infty$, or $\rho_{m} \rightarrow-\infty$ uniformly as $h_{j} \rightarrow \infty$, by Lemma (2.19), and so the map $f: \mathscr{H}\left(m_{j}\right) \rightarrow \mathbb{R}^{+}$defined by $f\left(h_{j}\right)=\operatorname{tr}\left(M^{2}\right)$ is again proper, and $f$ has a minimum.

Again, we show that the differential of $M\left(h_{j}\right)$ is nowhere singular. Let $\tilde{\alpha}=\tilde{\alpha}^{*}$, and make, as above, an infinitesimal gauge transformation $g=1+s x, x$ self adjoint, preserving the real equation; we have

$$
\frac{d}{d s}(M)=2 \Delta\left(x^{\prime}\right)-x u u^{*}-u u^{*} x-x w w^{*}-w w^{*} x .
$$


This will be nonzero if $x$ is nonzero, by the infinitesimal version of the argument given above; if one conjugates so that $x=\operatorname{diag}\left(t_{i}\right)$ then one has for the diagonal terms of $d M / d s$ :

$$
\frac{d}{d s}(M)_{i i}=2 \Delta\left(t_{i}^{\prime}\right)-2 t_{i} u_{i} \bar{u}_{i}-2 t_{i} w_{i} \bar{w}_{i}
$$

If $t_{1} \leqq \cdots \leqq t_{m}$, then, at $\mu_{j}, \Delta\left(t_{m}^{\prime}\right)<-K t_{m}, \Delta\left(t_{1}^{\prime}\right)>-K t_{1}$ for some positive $K$, by the infinitesimal version of (2.19), and so $|(d / d s)(M)|>c|x|$, for some positive $c$.

As in the preceding proposition, there is then a unique $h_{j}$ for which $M\left(h_{j}\right)=0$.

Combining (2.20) (2.21), one has by induction that there is on $\left(\mu_{1}, \mu_{N}\right)$, a set of solutions parametrized by $\mathscr{H}\left(r_{1}\right) \times \mathscr{H}\left(r_{N}\right)$, i.e., a point.

Theorem (2.22). Each G-equivalence class of Nahm complexes contains a unique $\mathscr{G}_{\mathbb{R}}$ equivalence class of real Nahm complexes.

Remark. It is perhaps appropriate here to insert a remark about the continuity of this procedure, i.e., if $(\alpha, \beta, v)(t)$ is a continuous family (in some appropriate sense) of Nahm complexes, is the procedure which assigns to $(\alpha, \beta, v)(t)$ the corresponding real complex $\left(\alpha^{\prime}, \beta^{\prime}, v^{\prime}\right)(t)$ continuous? The answer is yes, and the reason is to be found in Lemma (2.4). Suppose that $(\alpha, \beta, v)(0)=\left(\alpha^{\prime}, \beta^{\prime}, v^{\prime}\right)(0)$; then $F((\alpha, \beta)(t))$ is small for small $t$, and using lemma (2.4), one finds that fixing $h\left(\mu_{1}\right)=h\left(\mu_{N}\right)=1$ the $h$ corresponding to the gauge transformation sending $(\alpha, \beta, v)(t)$ to $\left(\alpha^{\prime}, \beta^{\prime}, v^{\prime}\right)(t)$ is then also small.

\section{Nahm Complexes and Rational Maps}

We will start by giving a sheaf theoretic formulation of a based rational map $F: \mathbb{P}_{1} \rightarrow S U(N) / T$ suited to our purposes. As is usual, in what follows, we denote by the same symbol a vector bundle and its sheaf of sections. Let $E$ be the trivial rank $n$ bundle over $\mathbb{P}_{1}$, with a fixed global basis $\left\{e_{1}, \ldots, e_{N}\right\}$. One can define a standard flag of subbundles:

$$
E_{0}^{+}=\{0\}, \quad E_{1}^{+}=\left\langle e_{1}\right\rangle, \quad E_{2}^{+}=\left\langle e_{1}, e_{2}\right\rangle, \ldots, E_{N}^{+}=E .
$$

Let $\bar{E}_{i}^{+}$denote the "anti-standard" flag:

$$
\bar{E}_{i}^{+}=\left\langle e_{N}, e_{N-1}, \ldots, e_{N-i+1}\right\rangle .
$$

A map $F: \mathbb{P}_{1} \rightarrow S U(N) / T$ can then be thought of as a flag $E_{1}^{-} \subset E_{2}^{-} \subset \cdots$ $\subset E_{N-1}^{-} \subset E$ of subbundles of $E$. We "base" the map $F$ by asking that $E_{i}^{-}$coincide with $\bar{E}_{i}^{+}$at $\infty$, i.e., $F(\infty)=\bar{E}_{i}^{+}$.

If the map $F$ is of degree $m=\left(m_{1}, \ldots, m_{N-1}\right)$, one has that $E_{i}^{-} / E_{i-1}^{-}$is the line bundle $\mathcal{O}\left(k_{N-i+1}\right)$, where $k_{i}=\left(m_{i}-m_{i-1}\right)$ (see, e.g., $\left.[M]\right)\left(m_{0}=m_{N}=0\right)$; on the other hand, $E_{i}^{+} / E_{i-1}^{+} \cong \mathcal{O}$. Now consider the sum $E_{i}^{+}+E_{N-i}^{-}$; except over a finite set of points, $E_{i}^{+}+E_{N-i}^{-}=E$, and so the sheaf

$$
Q_{i} \stackrel{\text { def }}{=} E /\left(E_{i}^{+}+E_{N-i}^{-}\right)
$$

is supported on a finite set of points. Similarly, $E_{i-1}^{+}+E_{N-i}^{-}$is of dimension $(N-1)$, 
anywhere where either $E_{i}^{+}+E_{N-i}^{-}$or $E_{i-1}^{+}+E_{N-i+1}^{-}$is of dimension $N$, i.e., away from the intersection of the supports of $Q_{i}$ and $Q_{i-1}$. In other words, the sheaf

$$
P_{i} \stackrel{\text { def }}{=} E /\left(E_{i-1}^{+}+E_{N-i}^{-}\right)
$$

is a line bundle away from $\operatorname{supp}\left(Q_{i}\right) \cap \operatorname{supp}\left(Q_{i-1}\right)$. Furthermore, one has exact sequences

$$
\begin{gathered}
0 \rightarrow \mathcal{O} \rightarrow P_{i} \stackrel{\pi_{i}}{\longrightarrow} Q_{i} \rightarrow 0, \\
0 \rightarrow \mathcal{O}\left(k_{i}\right) \rightarrow P_{i} \stackrel{\rho_{i}}{\rightarrow} Q_{i-1} \rightarrow 0,
\end{gathered}
$$

for $i=1, \ldots, N$. Note that $Q_{0}=Q_{N}=0$. One has an exact sequence (see [HuM, Proposition 1.12])

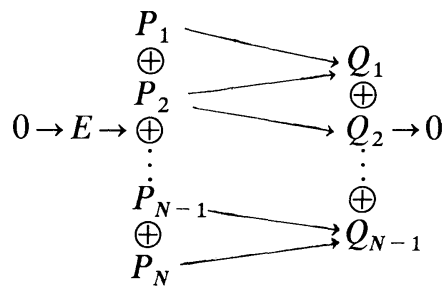

where the map between the last two terms is $\left(p_{1} \cdots p_{N}\right) \mapsto\left(\pi_{1}\left(p_{1}\right)-\rho_{2}\left(p_{2}\right)\right.$, $\left.\pi_{2}\left(p_{2}\right)-\rho_{3}\left(p_{3}\right), \ldots, \pi_{N-1}\left(p_{N-1}\right)-\rho_{N}\left(p_{N}\right)\right)$. Embedding $E$ in the center term, $E_{i}^{+}$is composed of sections of the form $\left(s_{1} \cdots s_{i}, 0 \cdots 0\right)$ and $E_{i}^{-}$of sections of the form $\left(0, \ldots, 0, t_{N-i+1}, \ldots, t_{N}\right)$.

As $Q_{i}$ is supported on points, $h^{1}\left(\mathbb{P}_{1}, Q_{i}\right)=0$, and so, as the space of rational maps of fixed degree is connected, $h^{0}\left(\mathbb{P}_{1}, Q_{i}\right)=h^{0}-h^{1}$ is a constant. For the generic rational map $[G], \operatorname{supp}\left(Q_{i}\right)$ is $m_{i}$ points, over each of which $Q_{i}$ is the skyscraper sheaf $\mathbb{C}$.

Therefore,

$$
h^{0}\left(\mathbb{P}_{1}, Q_{i}\right)=m_{i}
$$

(One also has that generically, $P_{i} \cong \mathcal{O}\left(m_{i}\right)$ )

Proposition (3.5). There is a natural equivalence between

- Based rational maps

$$
F: \mathbb{P}_{1} \rightarrow S U(N) / T
$$

-Equivalence classes under automorphisms of pairs $(S, e)$, where $S$ is a sequence of 0 -modules of the form (3.3) with:

$-Q_{i}$ supported over a finite set of points which doesn't include $\infty$,

$-h^{0}\left(\mathbb{P}_{1}, Q_{i}\right)=m_{i}$,

$-P_{i}, Q$ fitting into exact sequences (3.1), (3.2),

and $e=\left\{e_{1}, \ldots, e_{n}\right\}$ is a basis of $E$ at $\infty$ with $e_{i} \in P_{i}$. 
Proof. We have shown how, from a map $F$, one can obtain a pair $(S, e)$; we now show how to invert this procedure. We first prove that $E$ is locally free, and trivial. Define sections $s_{i}$ of $E$ of the form $\left(s_{i 1}, \ldots, s_{i, i-1}, 1,0, \ldots, 0\right)$, where 1 represents the section of $\mathcal{O}=\operatorname{ker}\left(\pi_{i}: P_{i} \rightarrow Q_{i}\right)$ which is equal to $e_{i}$ at $\infty$. Such sections exist, by the surjectivity of $P_{i} \rightarrow Q_{i}, P_{i} \rightarrow Q_{i-1}$; starting with the section of $\mathcal{O}$, one "zigzags up" the sequence (3.3). Let $r=\left(r_{1}, \ldots, r_{N}\right)$ be any other section, local or global of $E$. The last term, $r_{N}$, is a section of $\mathcal{O}=P_{N}$, and so there is a function $t_{N}$ such that $r-t_{N} s_{N}$ is of the form $\left(r_{1}^{\prime}, \ldots, r_{N-1}^{\prime}, 0\right), r_{N-1}^{\prime}$ is now a section of $\operatorname{ker}\left(\pi_{N-1}\right) \cong \mathcal{O}$, and so there is a function $t_{N-1}$ with $r-t_{N} s_{N}-t_{N-1} s_{N-1}$ of the form $\left(r_{1}^{\prime \prime}, \ldots, r_{N-2}^{\prime \prime}, 0,0\right)$. Iterating this procedure, one has $r=\sum t_{i} s_{i}$; if $r$ is a global section, the $t_{i}$ 's are constants; $E$ is a trivial bundle, with preferred basis $s_{i}$.

One then defines the subsheaf $E_{i}^{-}$as the subsheaf of $E$ of sections of the form $\left(0, \ldots, 0, u_{N-i+1}, \ldots, u_{N}\right)$. Near $\infty$, and in fact away from the support of the $Q_{i}, E_{i}^{-}$ is obviously locally free. On the other hand, away from $\infty$ one can choose trivializations of $\mathcal{O}\left(k_{i}\right)=\operatorname{ker} \pi_{i}$; with respect to these trivializations, define local sections $w_{i}$ of $E$ of the form $\left(0, \ldots, 0,1, w_{i, N-i+2}, \ldots, w_{i, N}\right)$, using the same zigzag procedure as above, but moving downward. $\left(w_{1}, \ldots, w_{i}\right)$ then forms a basis away from $\infty$ for $E_{i}^{-}$, which is then locally free; this is proven by the same procedure as above.

There remains to show that the $E_{i}^{-}$'s embed in $E$ as sub-bundles. This is equivalent to showing that $E / E_{i}^{-}$is locally free. However, this quotient is given by

$$
0 \rightarrow E / E_{i}^{-} \rightarrow \bigoplus_{i=1}^{N-i} P_{i} \rightarrow \bigoplus_{i=1}^{N-i-1} Q_{i} \rightarrow 0
$$

and so one uses the same techniques as above to show that $E / E_{i}^{-}$is indeed locally free.

One can use this to prove

Theorem (3.6). There is a natural bijective correspondence between and

- based rational maps $\mathbb{P}_{1} \rightarrow S U(N) / T$

-G-equivalence classes of Nahm complexes $(\alpha, \beta, v)$.

Proof. A) We begin by giving the map associated to a Nahm complex. Following (1.15), one remarks that on the interior of each interval $\left(\mu_{j}, \mu_{j+1}\right)$, the only invariant is the conjugacy class of $\beta_{j}$. An equivalent datum is the sheaf $Q_{j}$ defined by the sequence

$$
0 \rightarrow \mathcal{O}(-1)^{\oplus m_{j}} \stackrel{\left(\eta \llbracket-\beta_{j}(u)\right)}{\longrightarrow} \mathcal{O}^{\oplus m_{j}} \rightarrow Q_{j} \rightarrow 0 .
$$

Here $\eta$ is the standard coordinate on $\mathbb{P}_{1}$, and $u$ is a point in $\left(\mu_{j}, \mu_{j+1}\right) . Q_{j}$ is invariant under conjugation of $\beta_{j}$; it is supported over the eigenvalues of $\beta_{j}$, and its isomorphism class at an eigenvalue determines the Jordan form of $\beta_{j}$. For example, for a double eigenvalue $a$, the diagonal Jordan form corresponds to $(\mathcal{O} /\langle\eta-a\rangle)^{\oplus 2}$, while the non-diagonal Jordan form corresponds to $\mathcal{O} /\left\langle(\eta-a)^{2}\right\rangle$. 
In any case, from the sequence (3.7), one sees that

$$
H^{0}\left(\mathbb{P}_{1}, Q_{j}\right) \cong H^{0}\left(\mathbb{P}_{1}, \mathcal{O}^{\oplus m_{j}}\right) \cong \mathbb{C}^{m_{j}}
$$

The definition of the $P_{j}$ is obtained from the behavior of the Nahm complex near a boundary point $\mu_{j}$. We distinguish three cases:

1. $m_{j}>m_{j-1}$ :

The normal form proposition (1.15) tells us that $\beta_{j-1}$ can be taken to be a constant near $\mu_{j}$ and that $\beta_{j}$ is conjugate to a matrix with block form:

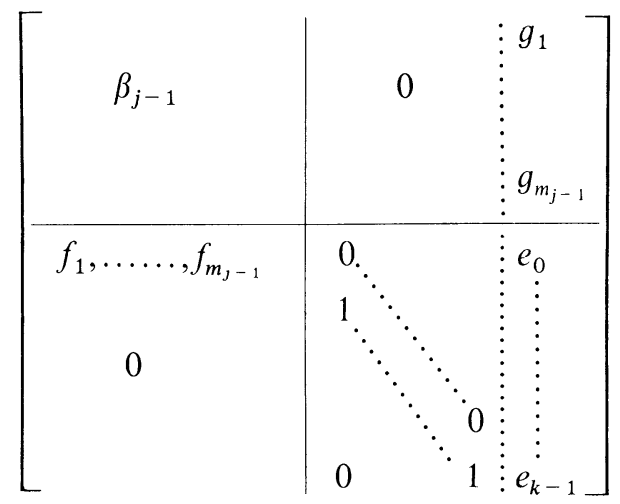

with $v_{j}$ the $\left(m_{j-1}+1\right)$ 'th basis vector. This form is determined up to the action of $G l\left(m_{j-1}, \mathbb{C}\right)$, where $s\left(\beta_{j-1}, f, g\right)=\left(s \beta_{j-1} s^{-1}, f s^{-1}, s g\right)$. One computes that:

$\operatorname{det}\left(\eta \mathbb{1}-\beta_{j}\right)=\operatorname{det}\left(\eta \mathbb{1}-\beta_{j-1}\right)\left(\eta^{k}-e_{k-1} \eta^{k-1}-\cdots-e_{0}\right)-f\left(\eta \mathbb{1}-\beta_{j-1}\right)_{\mathrm{adj}} \cdot g$,

where adj denotes the classical adjoint (matrix of cofactors). The $e_{i}$ 's are thus determined by the spectrum of $\beta_{j}$ and by $\beta_{j-1}, f$, and $g$. Let $\pi$ denote the projection onto the first $m_{j-1}$ coordinates. We define $P_{j}$ and the maps $P_{j} \rightarrow Q_{j}, P_{j} \rightarrow Q_{j-1}$ by the commuting diagram with exact rows:

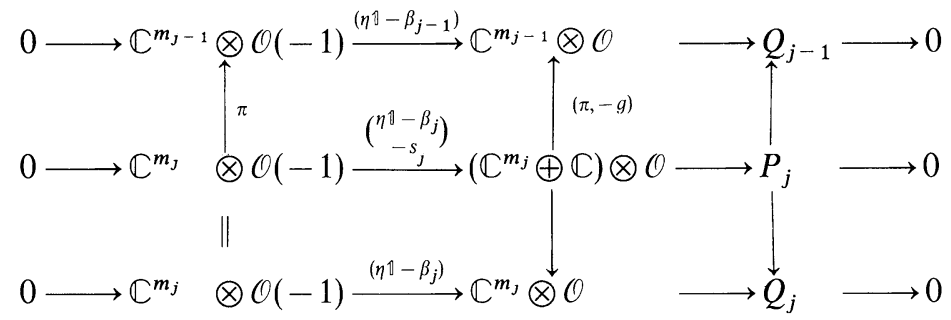

Here $s_{j}: \mathbb{C}^{m_{j}} \rightarrow \mathbb{C}$ is defined by $s_{j}=0$ on $\mathbb{C}^{m_{j-1}} \subset \mathbb{C}^{m_{j}}$, and $s_{j}\left(\beta_{j}^{n}\left(v_{j}\right)\right)=$ $\delta_{n, k-1},\left(k=m_{j}-m_{j-1}\right)$; in the basis used above, $s_{j}=(0, \ldots, 0,1)$.

This definition is $G l\left(m_{j-1}, \mathbb{C}\right)$-invariant. From (3.10) one easily sees that $P_{j}$ maps surjectively to $Q_{j}$, with kernel $\mathcal{O}$, proving (3.1); as for the map $P_{j} \rightarrow Q_{j-1}$, it is also surjective. To compute its kernel, let $i: \mathbb{C}^{m_{j-1}} \rightarrow \mathbb{C}^{m_{j}}$ be the injection into the first $m_{j-1}$ coordinates, and let $i_{v}: \mathbb{C} \rightarrow \mathbb{C}^{m_{j}}$ map 1 to $v_{j}$. Then one has the diagram, 
with exact rows, defining a sheaf $R_{j}$ and maps $R_{j} \rightarrow Q_{j-1}, R_{j} \rightarrow P_{j}$ :

$$
\begin{aligned}
& 0 \longrightarrow \mathbb{C}^{m_{j-1}} \otimes \mathcal{O}(-1) \stackrel{\left(\eta 1-\beta_{j-1}\right)}{\longrightarrow} \mathbb{C}^{m_{j-1}} \otimes \mathcal{O} \longrightarrow Q_{j-1} \longrightarrow 0 \\
& \begin{array}{c}
\| \\
0 \longrightarrow \mathbb{C}^{m_{j-1}} \otimes \mathcal{O}(-1) \stackrel{\left(\begin{array}{c}
\eta \uparrow-\beta_{j-1} \\
-f
\end{array}\right)}{\longrightarrow}\left(\mathbb{C}^{m_{j-1}} \oplus \mathbb{C}\right) \otimes \mathcal{O} \longrightarrow R_{j} \longrightarrow 0
\end{array}
\end{aligned}
$$

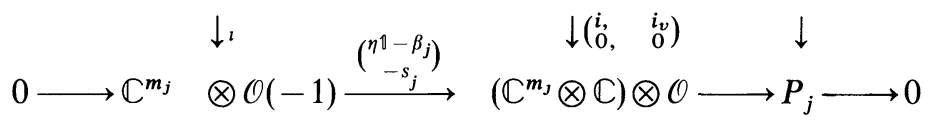

$R_{j}$ injects into $P_{j}$. Let $t(\eta): \mathbb{C}^{m_{j}} \rightarrow \mathbb{C}$ be defined by $t(\eta)\left(a_{1}, \ldots, a_{m_{j-1}}, b_{1} \cdots b_{k}\right)=$ $\eta^{k-1} b_{k}+\cdots+\eta b_{2}+b_{1}$, and let $p$ be the polynomial $\left(\eta^{k}-e_{k-1} \eta^{k-1} \cdots-e_{0}\right)$. Then one has a commuting diagram, away from $\eta=\infty$ :

$$
\begin{aligned}
& 0 \rightarrow \mathbb{C}^{m_{j}} \otimes \mathcal{O}(-1) \stackrel{\left(\begin{array}{c}
\eta \mathfrak{0}-\beta_{j} \\
-s_{j}
\end{array}\right)}{\longrightarrow}\left(\mathbb{C}^{m_{j}} \oplus \mathbb{C}\right) \otimes \mathcal{O} \quad \longrightarrow P_{j} \longrightarrow 0
\end{aligned}
$$

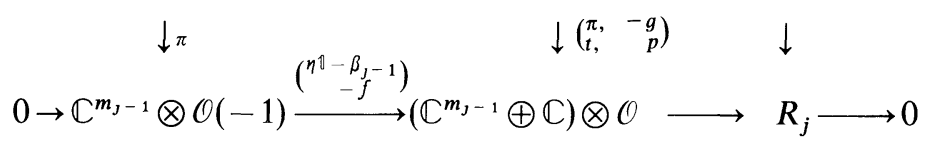

which defines away from $\eta=\infty$ a left inverse for the map $R_{j} \rightarrow P_{j}$ of (3.11). The map $P_{j} \rightarrow R_{j}$ has a pole of order $k$ at infinity, and so $R_{j} \cong P_{j}(-k)$. One now notes that the map $R_{j} \rightarrow Q_{j-1}$ of (3.11) is surjective, with kernel $\mathcal{O}$; however this map factors through $P_{j}$, showing that $P_{j}$ satisfies (3.2).

The basis of $P_{j}$ one chooses at $\infty$ is that defined by the section $(0,1)$ of $\left(\mathbb{C}^{m_{j}} \oplus \mathbb{C}\right) \otimes \mathcal{O}$.

2. $m_{j-1}>m_{j}$ :

One proceeds as above, with the following modifications: one changes all the $j$ indices to $(j-1)$, and all the $(j-1)$ indices to $j$; one then replaces $P_{j-1}$ by $R_{j}$, and $R_{j-1}$ by $P_{j}$.

3. $m_{j-1}=m_{j}$ :

One can gauge the Nahm complex to constant matrices $\beta_{j}, \beta_{j-1}$, with $\beta_{j}=\beta_{j-1}+u w^{T}$. One then defines $P_{j}$, and the maps by the commuting diagram:

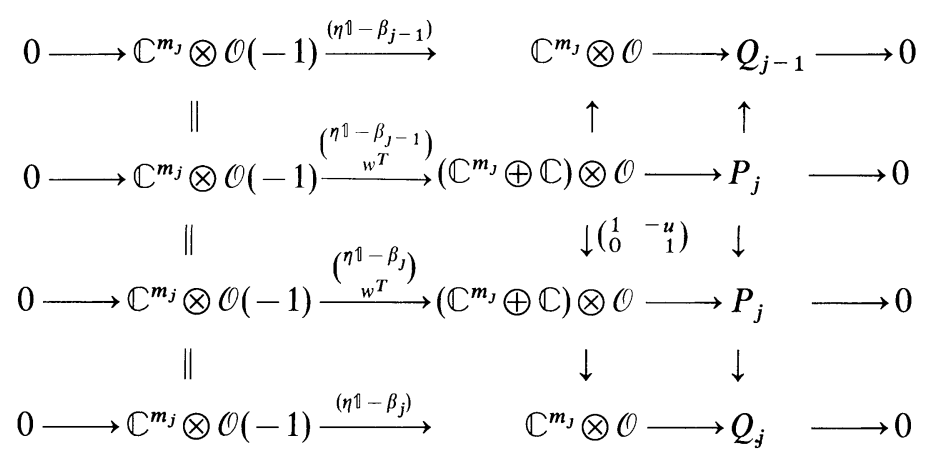

It is trivial to check that (3.1) and (3.2) are satisfied. Again, the trivialization one chooses at infinity is that given by the vector $(0,1) \in \mathbb{C}^{m_{j}} \oplus \mathbb{C}$. 
B) Suppose we are given the sheaf $P_{j}, Q_{j}$ corresponding to a rational map. We want to invert the procedure outlined above, and obtain a Nahm complex. The first step is to obtain the matrices $\beta_{j}$ up to conjugacy. To do this, one notes that as $Q_{j}$ has support not intersecting $\eta=\infty$, there is a well defined multiplication map $\times \eta: Q_{j} \rightarrow Q_{j}$. We define $\beta_{j}$ to be the induced endomorphism of $H^{0}\left(\mathbb{P}_{1}, Q_{j}\right)$.

From the sheaves $P_{j}$, and the maps $P_{j} \rightarrow Q_{j}, P_{j} \rightarrow Q_{j-1}$, we will now obtain the boundary data of a Nahm complex. To begin, as $P_{j}$ is a line bundle near $\infty$, one has a natural identification of $P_{j}(-l), l>0$, as sections of $P_{j}$ vanishing to order $l$ at $\infty$; for $l<0$, as sections with poles of order $-l$.

There are therefore sequences

$$
\begin{gathered}
0 \rightarrow \mathcal{O}(-l) \rightarrow P_{j}(-l) \rightarrow Q_{j} \rightarrow 0, \\
0 \rightarrow \mathcal{O}\left(k_{j}-l\right) \rightarrow P_{j}(-l) \rightarrow Q_{j-1} \rightarrow 0 .
\end{gathered}
$$

Also, as we have a fixed coordinate $\eta$, the trivializations of $P_{j}$ at $\infty$ induce trivializations of $P_{j}(-l)$.

We define, using (3.14),

$$
\begin{gathered}
H^{0}\left(\mathbb{P}_{1}, P_{j}(-1)\right) \cong H^{0}\left(\mathbb{P}_{1}, Q_{j}\right) \stackrel{\text { def }}{\cong} W_{j}=\mathbb{C}^{m_{j}}, \\
H^{0}\left(\mathbb{P}_{1}, P_{j}\left(-k_{j}-1\right)\right) \cong H^{0}\left(\mathbb{P}_{1}, Q_{j-1}\right) \stackrel{\text { def }}{\cong} V_{j}=\mathbb{C}^{m_{j-1}} .
\end{gathered}
$$

We distinguish three cases:

1. $m_{j}>m_{j-1}$ :

One has a natural inclusion $i: V_{j} \rightarrow W_{j}$, as sections vanishing to order $\left(-k_{j}-1\right)$. There is also a natural projection $\pi: W_{j} \rightarrow V_{j}$ defined by $H^{0}\left(\mathbb{P}_{1}, P_{j}(-1)\right) \rightarrow$ $H^{0}\left(\mathbb{P}_{1}, Q_{j-1}\right) ; \pi \circ i=\mathrm{Id}$. We can decompose $W_{j}$ as $i\left(V_{j}\right) \oplus \operatorname{ker}(\pi)$.

From the sequence $0 \rightarrow \mathcal{O} \rightarrow P_{j}\left(-k_{j}\right) \rightarrow Q_{j-1} \rightarrow 0$, one deduces that there is a unique section $v_{j}$ of $P_{j}$, vanishing to order $k_{j}$ at $\infty$, which lies in $\operatorname{ker}(\pi)$ and which coincides with the basis of $P_{j}(-k)$ at $\infty$. One then has a decomposition of $W_{j}$ as:

$$
W_{j} \cong V_{j} \oplus\left\langle v_{j}\right\rangle \oplus\left\langle\eta v_{j}\right\rangle \oplus \cdots \oplus\left\langle\eta^{k_{j-1}} v_{j}\right\rangle .
$$

Now consider the map $\times \eta: H^{0}\left(\mathbb{P}_{1}, Q_{j}\right) \rightarrow H^{0}\left(\mathbb{P}_{1}, Q_{j}\right) \cong W_{j} . W_{j}$ also decomposes as $H^{0}\left(\mathbb{P}_{1}, P_{j}(-2)\right) \oplus\left\langle\eta^{k-1} v_{j}\right\rangle$. On the first summand, multiplication by $\eta$ is just that induced by $\times \eta: P_{j}(-2) \rightarrow P_{j}(-1)$; on the other hand, for $\left\langle\eta^{k-1} v_{j}\right\rangle$, one must really evaluate in $Q_{j}$, multiply by $\eta$, then take the corresponding section. In a basis given by the decomposition (3.15), multiplication by $\eta$ has the form:

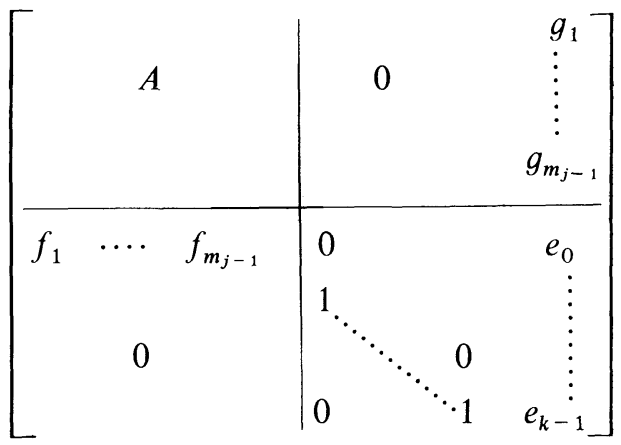


Furthermore, by comparing the operations

and

$$
\times \eta: H^{0}\left(\mathbb{P}_{1}, Q_{j-1}\right) \rightarrow H^{0}\left(\mathbb{P}_{1}, Q_{j-1}\right)
$$

$$
\times \eta: H^{0}\left(\mathbb{P}_{1}, P_{j}\left(-k_{j}-1\right)\right) \rightarrow H^{0}\left(\mathbb{P}_{1}, P_{j}\left(-k_{j}\right)\right),
$$

one can see that the block $A$ in the matrix above is precisely $\beta_{j-1}$.

2. $m_{j}<m_{j-1}$ :

One proceeds as above, inverting the roles of $V_{j}$ and $W_{j}$.

3. $m_{j}=m_{j-1}$ :

One has the sequences

$$
\begin{aligned}
& 0 \rightarrow \mathcal{O} \rightarrow P_{j} \rightarrow Q_{j} \rightarrow 0, \\
& 0 \rightarrow \mathcal{O} \rightarrow P_{j} \rightarrow Q_{j-1} \rightarrow 0 .
\end{aligned}
$$

Let $\rho$ be the section of $\mathcal{O}$ in (3.17), $\sigma$ be the section of $\mathcal{O}$ in (3.18), both taking as value at $\infty$ our basis element of $P_{j}$. One has:

$$
\begin{aligned}
H^{0}\left(\mathbb{P}_{1}, P_{j}(-1)\right) & \cong H^{0}\left(\mathbb{P}_{1}, Q_{j}\right) \cong H^{0}\left(\mathbb{P}_{1}, Q_{j-1}\right), \\
H^{0}\left(\mathbb{P}_{1}, P_{j}\right) & \cong H^{0}\left(\mathbb{P}_{1}, Q_{j-1}\right) \oplus\langle\sigma\rangle \\
& \cong H^{0}\left(\mathbb{P}_{1}, Q_{j}\right) \oplus\langle\rho\rangle .
\end{aligned}
$$

Consider the map induced on sections by $\times \eta: P_{j}(-1) \rightarrow P_{j}$; using the isomorphisms (3.19), (3.20), this is of the form, for $q \in H^{0}\left(\mathbb{P}_{1}, P_{j}(-1)\right)$.

$$
\eta q=\beta_{j-1} q+\sigma\left(w^{T} \cdot q\right)
$$

for some $w^{T}: H^{0}\left(\mathbb{P}_{1}, P_{j}(-1)\right) \rightarrow \mathbb{C}$; if, instead, one uses (3.19), (3.21), one gets

$$
\eta q=\beta_{j} \cdot q+\rho\left(\tilde{w}^{T} \cdot q\right) .
$$

As $\rho=\sigma$ at $\infty, \sigma-\rho=u \in H^{0}\left(\mathbb{P}_{1}, P_{j}(-1)\right)$, and so

$$
\beta_{j-1} \cdot q+u \cdot\left(w^{T} \cdot q\right)+\rho\left(w^{T} \cdot q\right)=\beta_{j} \cdot q+\rho\left(\tilde{w}^{T} \cdot q\right),
$$

which forces $w=\tilde{w}$, and $\beta_{j}=\beta_{j-1}+u w^{T}$. The matrices therefore have the correct rank one jump, and one puts $v_{j}=(u, w)$.

We have thus obtained

- a conjugacy class $\left[\beta_{j}\right]$ for each interval $\left(\mu_{j}, \mu_{j+1}\right)$;

- the correct data for a Nahm complex in normal form near each boundary point $\mu_{j}$ :

- the "extra data" $v_{j}$, for each $\mu_{j}$.

It is then straightforward to piece together the various "chunks" of Nahm complex defined near each $\mu_{j}$ via gauge transformations which are non-constant only on compact subsets of the $\left(\mu_{j}, \mu_{j+1}\right)$ 's, giving one a Nahm complex.

It is also easy to verify that the constructions of parts A) and B) of the proof are inverses of one another.

\section{Twistors and Monopoles}

We have shown that equivalence classes of pairs (solutions to Nahm's equations, extra data $v$ ) correspond naturally to based rational maps. This section addresses 
itself to two remaining problems. One is that of the meaning of the rational map in terms of the monopole; the other is the related problem of interpreting the extra data $v$. These questions have already been answered in the case of $S U(2)$-monopoles $[\mathrm{Hu}],[\mathrm{AHi}]$, and, as the answer here is essentially the same, we will be brief.

We first recall from $[M]$ the twistor transform for $S U(N)$ monopoles. Let $(\nabla, \varphi)$ be an $S U(N)$ monopole over $\mathbb{R}^{3}$, with $(\nabla, \varphi)$ acting on a rank $N$ bundle $V$. One defines a rank $N$ bundle $E$ over $T \mathbb{P}_{1}=$ oriented lines in $\left.\mathbb{R}^{3}\right\}$ by $E_{l}=\left\{s \in \Gamma(l, v) \mid\left(\nabla_{u}-i \varphi\right) s=0\right\}$, where $u$ is a positive unit vector field along the line l. Let $r$ denote the radial coordinate in $\mathbb{R}^{3}$.

The boundary conditions of $(\nabla, \varphi)$ imply that one can define a full flag $E_{i}^{+}$of subbundles of $E$ by

$$
\left(E_{i}^{+}\right)_{l}=\left\{s \in E_{l} \mid s \text { bounded by } r^{k} e^{-\mu_{N-i+1} r} \text { for some } k \text {, as } r \rightarrow \infty\right\} .
$$

Similarly, one defines $E_{i}^{-}$by considering decay in the negative direction. Setting $P_{i}=E /\left(E_{i-1}^{+}+E_{N-i}^{-}\right), Q_{i}=E /\left(E_{i}^{+}+E_{N-i}^{-}\right)$, one obtains a sequence of the form (3.3), but over $T \mathbb{P}_{1}$.

Now one restricts this sequence to a fiber $\mathbb{C}_{0}$ of the projection $T \mathbb{P}_{1} \rightarrow \mathbb{P}_{1}$; this is tantamount to considering only the lines in a fixed direction in $\mathbb{R}^{3}$, and is what corresponds to the splitting $\mathbb{R}^{3} \cong \mathbb{C} \times \mathbb{R}$ of the main theorem. Over $\mathbb{C}_{0}, Q_{i}$ is supported over a finite set of points, and $h^{0}\left(\mathbb{C}_{0}, Q_{i}\right)=m_{i}$. Furthermore, if one refers to the construction of the solution to Nahm's equations of [HuM], one sees that it is an exact parallel of the construction of the Nahm complex from the rational map in Sect. 3. In other words, $E$ and the two flags of subbundles $E_{i}^{+}, E_{i}^{-}$, restricted to $\mathbb{C}_{0} \subset T \mathbb{P}_{1}$, are exactly the restriction to $\mathbb{C}_{0}=\mathbb{P}_{1} \backslash\{\infty\}$ of the flags involved in the rational map.

Referring to Sect. 3, (compare [AHi]), the meaning of the extra information $v$ associated to a solution to Nahm's equations is clear; in essence, $v$ determines a (unitary) trivialization $e_{i}$ of $E$ at $\infty$ in $\mathbb{P}_{1}$, such that $e_{i}$, at infinity, lies in $E_{i}^{+} \cap E_{N-i+1}^{-}$. Interpreting in terms of scattering data as in [AHi, Sect. 3], one has the following picture. One fixes a direction in $\mathbb{R}^{3}$, let us say the positive $z$-axis. The lines in this direction then correspond naturally to $(x+i y) \in \mathbb{C}$. Integrating $\left(\nabla_{z}+i \varphi\right)$, one obtains over $\mathbb{C}$ a bundle $E$ containing two flags $E_{i}^{+}, E_{i}^{-}$, which are defined by decay behaviour at $+\infty,-\infty$ respectively. The $v_{i}$ 's correspond to a choice of unitary trivialization of the bundle $E$ over $\mathbb{C}$ such that $E_{i}^{+}$is the standard flag, and such that $E_{i}^{-}$tends to the anti-standard flag as $|x+i y| \rightarrow \infty$; such a trivialization is defined from a trivialization of the bundle $V$ over $\mathbb{R}^{3}$, which is asymptotically flat as $z \rightarrow+\infty$. We refer to such a trivialization as a $v$-framing; for a given $(\nabla, \varphi)$, there is a torus $T^{N}$ of such framings. Given such a framing, a rational map is defined by the flag $E_{i}^{-}$, as in [AHi].

The moduli space $\tilde{M}_{m}$ we have studied is thus that of pairs (monopoles, $v$-framing). Its fiber over a point $(\nabla, \varphi)$ of the monopole moduli space is then the torus $T^{N}$, quotiented by the subgroup of $T^{N}$ which stabilizes $(\nabla, \varphi)$. When the monopole is irreducible, this stabilizer is simply the $S^{1}$ of unitary multiples of the identity; the fiber is then the torus $T^{N-1}$ of $S U(N)$. 


\section{The Cases of $S O(N)$ and $S p(K)$}

We treat the case of $S O(N)$ and $S p(K)$ monopoles by considering them as $S U(N)$ monopoles with extra structure. (For $S p$, we set $N=2 K$.) One then just retraces the main steps of the proof for the $S U(N)$ case, checking at each step what extra structure must be added.

A) Nahm's Equations and Nahm Complexes. One has [HuM] that for both SO, $S p$, the intervals on which one solves Nahm's equations are symmetric about the origin:

$$
\left(\mu_{N-j-1}, \mu_{N-j}\right)=\left(-\mu_{j+1},-\mu_{j}\right) .
$$

Let the solutions ${ }^{i} T_{j}$ to Nahm's equations act on vector spaces $V_{j}$; one has the extra structure of a nondegenerate pairing

$$
c_{j}: V_{j} \rightarrow V_{N-j}^{*}
$$

with respect to which ${ }^{i} T_{j}(z),{ }^{i} T_{N-j}(-z)$ are adjoint, i.e.,

$$
c_{j}^{i} T_{j}(z) c_{j}^{-1}=\left({ }^{i} T_{N-j}(-z)\right)^{T} .
$$

The $c_{j}$ are compatible with the boundary gluing between the intervals, in the following sense:

- at a non-neutral boundary point $\mu_{j}$ one has a projection and an injection:

$$
\pi_{j} \cdot \mathbb{C}^{\bar{m}} \rightarrow \mathbb{C}^{m} ; \quad i_{j}: \mathbb{C}^{m} \rightarrow \mathbb{C}^{\bar{m}}
$$

The compatibility condition is then:

$$
\begin{aligned}
c_{j-1} \pi_{j} & =i_{N-j+1}^{T} c_{j}, & \text { for } \quad m_{j}>m_{j-1}, \\
c_{j} \pi_{j} & =i_{N-j+1}^{T} c_{j-1}, & \text { for } \quad m_{j}<m_{j-1}
\end{aligned}
$$

- at a neutral boundary point, one asks that $c_{j}=c_{j-1}$.

For $S O(N)$, one has the symmetry condition:

and for $S p(K)$ :

$$
c_{j}=-c_{N-j}^{T},
$$

$$
c_{j}=c_{N-j}^{T} \text {. }
$$

Turning now to Nahm complexes, one asks that

$$
\begin{aligned}
& c_{j} \alpha_{j} c_{j}^{-1}=\alpha_{N-j}^{T}, \\
& c_{j} \beta_{j} c_{j}^{-1}=\beta_{N-j}^{T}
\end{aligned}
$$

for the $v_{j}$, one imposes the condition that, at a non-neutral boundary point $\mu_{j}$, $j \leqq(N+1) / 2$ :

$$
\begin{gathered}
c\left(i_{j}\left(\mathbb{C}^{m}\right)\right)\left(v_{N-j+1}\right)=0, \\
c\left(\beta^{l} v_{j}\right)\left(v_{N-j+1}\right)=-\delta_{l, \bar{m}-\underline{m-1}} \text { for } l=0, \ldots, \bar{m}-\underline{m}-1
\end{gathered}
$$

and, at a neutral boundary point $\mu_{j}, j \leqq(N+1) / 2$, if $v_{j}=(u, w), v_{N-j+1}=\left(u^{\prime}, w^{\prime}\right)$,

$$
c_{j}(u)=-w^{\prime} .
$$


One constrains the gauge transformations by

$$
g(-z)^{-1^{T}}=c g(z) c^{-1}
$$

these are the transformations keeping $c_{j}$ constant.

The proof that for each equivalence class of Nahm complexes one has an essentially unique solution proceeds exactly as before; the unique solution must then be invariant under $c$. In fact, this case is already considered by Donaldson, who treats $S U(2)$ as $S p(1)$.

B) Nahm Complexes and Rational Maps. One wants an equivalence between Nahm complexes and rational maps into flag manifolds for $S O(N)$ and $S p(K)$. The flag manifolds one must consider are those of full flags $E_{1} \subset E_{2} \subset \cdots \subset E_{N}=\mathbb{C}^{N}$ satisfying $E_{N-i}=E_{i}^{\perp}$ with respect to some standard quadratic form. We call these flags isotropic-coisotropic. For $S O(N)$, the quadratic form is taken to be

$$
\left\langle\left(a_{i}\right),\left(b_{i}\right)\right\rangle=\sum_{i=1}^{N} a_{i} b_{N-i+1}
$$

and, for $S p(K)$

$$
\left\langle\left(a_{i}\right),\left(b_{i}\right)\right\rangle=\sum_{i=1}^{K} a_{i} b_{N-i+1}-b_{i} a_{N-i+1} .
$$

We will show that, given a $c$-invariant $\mathrm{Nahm}$ complex $(\alpha, \beta, v)$, the rational map one obtains by the procedure of Sect. 3 is indeed into the submanifold of isotropic-coisotropic flags. To do this, we proceed in several steps, using the sequence $0 \rightarrow E \rightarrow \oplus P_{i} \rightarrow \oplus Q_{i} \rightarrow 0$ of (3.3).

i) To begin, one notes that our pairing $c_{j}$ is invariantly a pairing $H^{0}\left(\mathbb{P}_{1}, Q_{j}\right) \otimes$ $H^{0}\left(\mathbb{P}_{1}, Q_{N-j}\right) \rightarrow \mathbb{C}$, with respect to which the adjoint of $\beta_{j}$ is $\beta_{N-j}$. From this, we will define a pairing

$$
H^{0}\left(\mathbb{P}_{1}, P_{j}\right) \otimes H^{0}\left(\mathbb{P}_{1}, P_{N-j+1}\right) \rightarrow H^{0}\left(\mathbb{P}_{1}, \mathcal{O}\left(m_{j}+m_{j-1}\right)\right),
$$

where $\mathcal{O}\left(m_{j}+m_{j-1}\right)$ is to be thought of as the sheaf of functions with poles over the support of $Q_{j}$ and $Q_{j-1}$.

ii) We then show that this pairing descends to one over $P_{j} \otimes P_{N-j-1}$.

iii) One then considers the induced pairing on local sections of $E \subset\left(\oplus P_{i}\right)$; one shows that for sections of $E$, the poles cancel, and one is left with a holomorphic pairing. One also checks that the induced pairing on global sections of $E$ is the standard one. The fact that, in $\left(\oplus P_{i}\right), P_{j}$ is only paired with $P_{N-j+1}$ then implies that the flags $E_{i}^{+}, E_{i}^{-}$defined in Sect. 3 are isotropic-coisotropic.

Let $G=S O(N)$ or $S p(K)$ be our group. We define

$$
\begin{aligned}
\tau_{j} & =-1 \text { if } j>N / 2, \text { and } G=S O(N) \\
& =1 \text { otherwise, } \\
\sigma_{j} & =-1 \text { if } j>K=N / 2, \text { and } G=S p(K) \\
& =1 \text { otherwise. }
\end{aligned}
$$

Let $\{,\}_{j}$ denote the pairing between sections of $Q_{j}$ and sections of $Q_{N-j}$ defined 
by $c_{j}$. We define the pairing of sections of $P_{j}$ with sections of $P_{N-j-1}$ by distinguishing three separate cases:

i) $m_{j}>m_{j-1}$

In this case, $P_{j}$ is defined by (3.10):

$$
0 \rightarrow \mathbb{C}^{m_{j}} \otimes \mathcal{O}(-1) \stackrel{\left(\begin{array}{c}
\eta \uparrow-\beta_{j} \\
-s_{j}
\end{array}\right)}{\longrightarrow}\left(\mathbb{C}^{m_{j}} \oplus \mathbb{C}\right) \otimes \mathcal{O} \rightarrow P_{j} \rightarrow 0
$$

and, as $m_{N-j}=m_{j}>m_{j-1}=m_{N-j+1}, P_{N-j+1}$ is defined by (3.11):

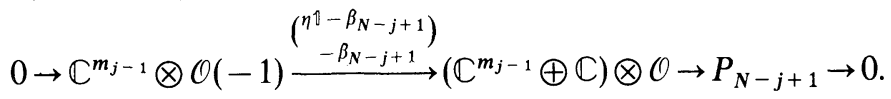

Let $\pi=\pi_{j}: \mathbb{C}^{m_{j}} \rightarrow \mathbb{C}^{m_{j-1}}$ be the projection, and $i=i_{N-j+1}: \mathbb{C}^{m_{j-1}} \rightarrow \mathbb{C}^{m_{j}}$ be the corresponding injection; set $v=v_{N-j+1}$. Referring to (3.8), we define a pairing between $(a, x) \in \mathbb{C}^{m_{j}} \oplus \mathbb{C} \cong H^{0}\left(\mathbb{P}_{1}, P_{j}\right)$ and $(b, y) \in \mathbb{C}^{m_{j-1}} \oplus \mathbb{C} \cong H^{0}\left(\mathbb{P}_{1}, P_{N-j+1}\right)$ by:

$$
\begin{aligned}
& ((a, x),(b, y))_{j}=\left\{\left(\eta \mathbb{1}-\beta_{j-1}\right)^{-1}\left(\pi(a)-g_{j} x\right), b\right\}_{j-1} \\
& -\left\{\left(\eta \mathbb{1}-\beta_{j}\right)^{-1} a, i(b)+i_{v}(y)\right\}_{j}+\tau_{j} x \cdot y .
\end{aligned}
$$

Here $\eta$ is the standard coordinate on $\mathbb{P}_{1}$. Now, if $\left.(a, x)=\left(\left(\eta \mathbb{1}-\beta_{j}\right) u,-s_{j} \cdot u\right)\right)$ one finds that

$$
((a, x),(b, y))_{j}=\{\pi(u), b\}_{j-1}-\left\{u, i(b)+y v_{N-j+1}\right\}_{j}-\tau_{j} s_{j}(u) \cdot y .
$$

Referring to (3.10) and (5.6), $s_{j}=-\tau_{j}\left\{\cdot, v_{N-j+1}\right\}_{j}$; also by the compatibility condition (5.2) $\pi$ is the adjoint of $i$. The r.h.s. of (5.15) therefore vanishes. Similarly, (5.14) also vanishes when $(b, y)=\left(\left(\eta 1-\beta_{N-j+1}\right) w,-f_{N-j+1} w\right)$. Referring to (5.12), (5.13), this means that the product $(,)_{j}$ descends to:

$$
(,)_{j}: P_{i} \otimes P_{N-j+1} \rightarrow \mathcal{O}\left(m_{j}+m_{j-1}\right) .
$$

ii) $m_{j}<m_{j-1}$ :

One proceeds in exactly symmetrical fashion; defining the pairing by:

$$
\begin{aligned}
((a, x),(b, y))_{j}= & \left\{\left(\eta \mathbb{1}-\beta_{j-1}\right)^{-1}\left(i(a)+i_{v}(x)\right), b\right\}_{j-1} \\
& -\left\{\left(\eta \mathbb{1}-\beta_{j}\right)^{-1} a, \pi(b)-g_{N-j+1} y\right\}_{j}-\tau_{j} x \cdot y
\end{aligned}
$$

iii) $m_{j-1}=m_{j}$ :

In this case, one has that $P_{j}, P_{N-j+1}$ are defined by (3.13),

$$
\begin{aligned}
& 0 \rightarrow \mathbb{C}^{m_{j}} \otimes \mathcal{O}(-1) \stackrel{\left({ }^{\eta 1-\beta_{j-1}}\right)}{\stackrel{w^{T}}{\longrightarrow}}\left(\mathbb{C}^{m_{j}} \oplus \mathbb{C}\right) \otimes \mathcal{O} \rightarrow P_{j} \rightarrow 0, \\
& 0 \rightarrow \mathbb{C}^{m_{j}} \otimes \mathcal{O}(-1) \stackrel{\left({ }^{\eta 1-\beta_{N-\jmath}}\right)}{\stackrel{w^{\prime} T}{\longrightarrow}}\left(\mathbb{C}^{m_{j}} \oplus \mathbb{C}\right) \otimes \mathcal{O} \rightarrow P_{N-j+1} \rightarrow 0,
\end{aligned}
$$

where $\beta_{j}=\beta_{j-1}+u w^{T}, \beta_{N-j+1}=\beta_{N-j}+u^{\prime} w^{\prime T}$. As above, one defines a pairing between sections of $P_{j}$ and $P_{N-j+1}$, by

$$
((a, x),(b, y))_{j}=\left\{\left(\eta \mathbb{1}-\beta_{j-1}\right)^{-1} a, b-u^{\prime} y\right\}_{j-1}-\left\{\left(\eta \mathbb{1}-\beta_{j}\right)^{-1}(a-u x), b\right\}_{j}+\sigma_{j} x y,
$$


again, one checks, using (5.7), that this descends to $P_{j} \otimes P_{N-j+1}$.

Summing one obtains a bilinear form, with poles, on $\oplus P_{i}$ defined by

$$
\left(\left(a_{i}\right),\left(b_{i}\right)\right)=\sum_{i=1}^{N}\left(a_{i}, b_{N-i+1}\right)_{i}
$$

One therefore has a pairing on local sections of $E$. Referring, however, to the sequence (3.3) which defines $E$, and to the definitions of the forms $(\cdot, \cdot)_{j}$, one easily sees that the poles cancel, giving a pairing $E \otimes E \rightarrow \mathcal{O}$. As remarked above, as the pairing on $E$ is a sum of pairings on $P_{i} \otimes P_{N-i+1}$, one automatically has that $E_{i}^{+}, E_{i}^{-}$are isotropic-coisotropic. To evaluate the pairing on sections of $E$, it suffices as $E$ is trivial, to evaluate at $\eta=\infty$. Referring to (5.14), (5.17), one sees that only the $\pm \tau_{j} x y, \sigma_{j} x y$ terms contribute at infinity and, with respect to the standard basis of $H^{0}\left(\mathbb{P}_{1}, E\right)$, the pairing is indeed the standard one. Also note that if $\{,\}_{j}= \pm\{,\}_{N-j}$, then $(,)_{j}=\mp(,)_{N-j+1}$; from (5.3), (5.4), the pairing on $E$ is indeed symmetric for $S O(N)$, antisymmetric for $S p(N)$.

The last thing to be checked is that one can invert the above procedure. One wants to obtain from an isotropic-coisotropic rational map, a $c$-invariant $\mathrm{Nahm}$ complex, i.e., a non-degenerate pairing

$$
\{,\}_{j}: H^{0}\left(\mathbb{P}_{1}, Q_{j}\right) \otimes H^{0}\left(\mathbb{P}_{1}, Q_{N-j}\right) \rightarrow \mathbb{C},
$$

such that (5.2) to (5.7) are satisfied.

To do this, we note that away from the support of the $Q_{i}, E \cong\left(\oplus P_{i}\right)$, and so pairing on $E$ defines a pairing $($,$) on sections of \left(\oplus P_{i}\right)$; this extends over all of $\mathbb{P}_{1}$ to a meromorphic pairing. As $E_{i}^{+}, E_{i}^{-}$are isotropic-coisotropic, one finds that

$$
\left(P_{j}, P_{k}\right) \neq 0 \text { only if } k=N-i+1 .
$$

Let $q$ be a section of $Q_{j}, q^{\prime}$ a section of $Q_{N-j}$. Then, over $\mathbb{P}_{1} \backslash\{\infty\}$, there is a section $p=\left(p_{1}, \ldots, p_{j}, 0 \ldots 0\right)$ in $\oplus P_{i}$ mapping to $(0 \ldots 0, q, 0 \ldots 0)$ in $\oplus Q_{i}$; similarly, one has a section $p^{\prime}=\left(0, \ldots, 0, p_{N-j+1}^{\prime}, \ldots, p_{N}^{\prime}\right)$ of $\otimes P_{i}$ mapping to $\left(0, \ldots, 0, q^{\prime}, 0 \ldots 0\right)$. Define

$$
\left\{q, q^{\prime}\right\}_{j}=-\sum_{\text {poles } \neq \infty} \operatorname{res}\left(p, p^{\prime}\right)
$$

To see that this is well defined, note that $p$ and $p^{\prime}$ are determined up to addition of sections of $E$. Suppose that $p$ is a section of $E$; then, referring to (3.3), $p^{\prime}=\left(0, \ldots, 0, p_{N-j+1}, \ldots, p_{N}\right)$ can be "completed" to $\tilde{p}^{\prime}=\left(p_{1}^{\prime}, \ldots, p_{N-j}^{\prime}, p_{N-j+1}^{\prime}, \ldots, p_{N}^{\prime}\right)$ a section of $E$, and, by $(5.18),\left(p, p^{\prime}\right)=\left(p, \tilde{p}^{\prime}\right)$; but this last expression stays finite as $p, \tilde{p}^{\prime}$ are sections of $E$. Similarly, if $p^{\prime}$ is a section of $E$, one completes $p$ to $\tilde{p}$, a section of $E$, and $\left(p, p^{\prime}\right)=\left(\tilde{p}, p^{\prime}\right)=$ finite.

In a similar vein, one shows that the pairing is non-degenerate. Suppose that $\left\{q, q^{\prime}\right\}_{j}=0$ for all $q^{\prime}$. This is equivalent to saying that $\left(p, p^{\prime}\right)$ stays finite, for all sections $p^{\prime}=\left(0, \ldots, 0, p_{N-j+1}^{\prime}, \ldots, p_{N}^{\prime}\right)$ mapping to $\left(0, \ldots, 0, Q_{N-j}, 0 \ldots 0\right)$; "completing," this is the same as $\left(p, \check{p}^{\prime}\right)$ finite, for all $\tilde{p}^{\prime}$ sections of $E$. However, $p$ already represents a meromorphic section of $E$; if $\left(p, \tilde{p}^{\prime}\right)$ stays finite for all $\tilde{p}^{\prime}$, then $p$ is holomorphic, and its image $q \in Q_{j}$ is then zero.

That $\beta_{j}, \beta_{N-j}$ are adjoints of one another follows from the fact that they are 
defined as the maps induced on global sections by the multiplication map $\times \eta$ of $Q_{j}, Q_{N-j}$. For $\alpha_{N-j}, \alpha_{j}$, one uses the fact that the pairing respects the trivializations of the bundles over $\left(\mu_{j}, \mu_{j+1}\right),\left(\mu_{N-j-1}, \mu_{N-j}\right)$ with respect to which the $\alpha$ 's are zero. The proof of the rest of relations (5.2) to (5.7) follows from the definition, and the fact that this construction inverts that given above is also straightforward.

Acknowledgements. The author would like to thank N. P. Buchdahl, P. B. Kronheimer, and G. Matić for useful discussions and would also like to express his gratitude to the Institute for Advanced Study for their hospitality and support.

\section{References}

[Ahi] Atiyah, M. F., Hitchin, N. J.: The geometry and dynamics of magnetic monopoles. Princeton, NJ: P.U.P. 1988

[B] Buchdahl, N. P.: Hermitian-Einstein connections and stable vector bundles over compact complex surfaces. Math. Ann. (to appear)

[D1] Donaldson, S. K.: Nahm's Equations and the classification of monopoles. Commun. Math. Phys. 96, 387-407 (1988)

[D2] Donaldson, S. K.: Anti-self-dual Yang Mills connections over complex algebraic surfaces and stable vector bundles. Proc. Lond. Math. Soc. 50, 1-26 (1985)

[D3] Donaldson, S. K.: Infinite determinants, stable bundles and curvature. Duke Math. J. 54, 1, 231-247 (1987)

[G] Gravesen, J.: On the topology of spaces of holomorphic maps. Roskilde University preprint

[Ha] Hartman, P.: Ordinary differential equations. (Sec. Edn). Boston, MA: Birkhäuser 1982

[Hi] Hitchin, N. J.: On the construction of monopoles. Commun. Math. Phys. 89, 145-190 (1983)

[Hu] Hurtubise, J. C.: Monopoles and rational maps: A note on a theorem of donaldson. Commun. Math. Phys. 100, 191-196 (1985)

[HuM] Hurtubise, J. C., Murray, M. K.: On the construction of monopoles for the classical groups. Commun. Math. Phys. (to appear)

[JT] Jaffe, A., Taubes, C. H.: Vortices and monopoles. Boston, MA: Birkhäuser 1980

[M] Murray, M. K.: Non-Abelian magnetic monopoles. Commun. Math. Phys. 96, 539-565 (1984)

[N] Nahm, W.: All self-dual multi-monopoles for arbitrary gauge group. CERN Preprint TH-3172 (1981)

[T] Taubes, C. H.: Min-Max theory for the Yang-Mills-Higgs equations. Commun. Math. Phys. 97, 473-540 (1985)

[UY] Uhlenbeck, K. K., Yau, S.-T.: On the Existence of Hermitian-Yang-Mills connections in stable vector bundles. Commun. Pure Appl. Math. 39, 257-293 (1986)

Communicated by A. Jaffe

Received August 1, 1988 
